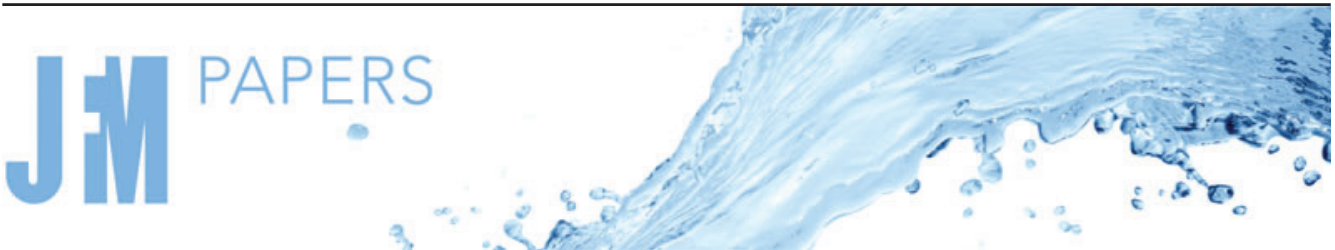

\section{The effect of inlet turbulence on the quiescent core of turbulent channel flow}

\author{
Masoud Asadi ${ }^{1}$, Md Kamruzzaman ${ }^{1}$ and R. Jason Hearst ${ }^{1}{ }^{\dagger}$ \\ ${ }^{1}$ Department of Energy and Process Engineering, Norwegian University of Science and Technology, \\ Trondheim NO-7491, Norway
}

(Received 14 June 2021; revised 1 November 2021; accepted 24 December 2021)

The impact of inlet turbulence on the structure of turbulent channel flow is investigated using particle image velocimetry. Streamwise-wall-normal plane measurements are performed in a channel, where different turbulence intensities were generated at the inlet with an active grid. Four cases are tested with matched centreline mean velocities, while the centreline turbulence intensities ranged from $3.7 \%$ for the reference case, up to $6.4 \%$. The friction velocity is found to be approximately constant with varying centreline turbulence intensities, resulting in a matched friction Reynolds number of $R e_{\tau} \approx 770$ for all cases, which contrasts with similar experiments performed in a zero-pressure-gradient boundary layer. The log region remains intact for all cases. The so-called quiescent core of the turbulent channel flow is also investigated. In addition to increased core discontinuity, the increased fluctuations of the streamwise velocity give rise to new core states, which differ from the conventional ones in their characteristic velocity. They are associated with a bulk of low- or high-momentum fluid passing through the measurement domain, and their occurrence increases with turbulence intensity. Tracking the core boundaries indicates an overall tendency of the core to move closer to the wall for increased inlet turbulence intensities, resulting in an increased core thickness. Moreover, it is found that the low-momentum cores generally reside closer to the wall compared with the ordinary cores and appear to be thicker than them, whereas the opposite, i.e. residing farther from the wall and being thinner, is true for the high-momentum cores.

Key words: boundary layer structure, turbulent boundary layers

\section{Introduction}

A fully developed channel flow is a canonical wall-bounded turbulent flow and has been of great interest because of its geometrical simplicity as well as pervasive applications

$†$ Email address for correspondence: jason.hearst@ntnu.no

(C) The Author(s), 2022. Published by Cambridge University Press. This is an Open Access article, distributed under the terms of the Creative Commons Attribution licence (https://creativecommons. org/licenses/by/4.0/), which permits unrestricted re-use, distribution, and reproduction in any medium, provided the original work is properly cited. 


\section{Asadi, M. Kamruzzaman and R. Jason Hearst}

in engineering. As a result, a large number of experimental (e.g. Johansson \& Alfredsson 1982; Wei \& Willmarth 1989; Monty et al. 2009; Schultz \& Flack 2013) and computational studies (e.g. Kim, Moin \& Moser 1987; Moser, Kim \& Mansour 1999; Hoyas \& Jiménez 2006; Bernardini, Pirozzoli \& Orlandi 2014; Lee \& Moser 2015) of channel flows have been carried out. Channel flows, together with pipe flows, constitute one of the two main categories of the wall-bounded turbulent flows, known as internal flows. The other category is external flows (Smits, McKeon \& Marusic 2011). From a conventional viewpoint, the internal flows are considered fully developed turbulent flows, in contrast to external flows where a turbulent boundary layer (TBL) is in contact with a non-turbulent potential region through an interface known as the turbulent/non-turbulent interface. This viewpoint was slightly altered by the identification of the quiescent core (QC), i.e. the highest uniform momentum zone (UMZ), of the turbulent channel flow by Kwon et al. (2014).

For the first time, Meinhart \& Adrian (1995) reported the presence of time-varying zones in a wall-bounded flow separated from one another by thin viscous shear layers. This was the starting point for researchers to investigate the so-called UMZs. Using particle image velocimetry (PIV), Adrian, Meinhart \& Tomkins (2000) explored the underlying features of UMZs in a zero-pressure-gradient turbulent boundary layer (ZPG-TBL) and proposed a method to identify them by assessing the histograms of the streamwise velocity component. They argued that each modal velocity, i.e. the peak in the histogram, corresponds to a UMZ. This method has been widely adopted in later studies to detect UMZs present in ZPG-TBLs (de Silva, Hutchins \& Marusic 2016; Laskari et al. 2018; Hearst et al. 2021; Laskari \& McKeon 2021) as well as turbulent channel (Kwon et al. 2014; Yang, Hwang \& Sung 2016; Jie et al. 2019) and pipe flows (Yang, Hwang \& Sung 2019; Chen, Chung \& Wan 2020; Gul, Elsinga \& Westerweel 2020). Recently, Fan et al. (2019) presented a new method to detect UMZ edges, arguing that it can overcome some of the main limitations of the histogram-based method, such as sensitivity to the bin size and peak detection parameters.

Kwon et al. (2014) performed PIV measurements over a large area of a fully developed turbulent channel flow. They devised a technique based on the histogram method (Adrian et al. 2000), which examines the probability density function (p.d.f.) of the accumulated modal velocities of all PIV fields. They found a UMZ which settled at the centre of the channel bounded by contour lines of a constant velocity threshold, equal to $95 \%$ of the centreline mean velocity (Yang et al. (2016), Jie et al. (2019) and Jie, Andersson \& Zhao (2021) found the same boundary threshold value in their studies). Kwon et al. (2014) called this UMZ the 'core' of the turbulent channel flow and referred to it as 'quiescent' because the streamwise velocity fluctuations were low throughout this region. Although the identified QCs were discontinuous sometimes $(8.7 \%-21.5 \%$ of the total PIV frames for different Reynolds numbers), they were present in all PIV frames. Some other features of a UMZ edge were also observed at the boundary of the QC, e.g. an abrupt jump in the streamwise velocity and a sharp peak in the spanwise vorticity. The authors examined the QCs for four different Reynolds numbers $\left(R e_{\tau}=1000,2000,3000\right.$ and 4000, where $R e_{\tau}$ is the friction Reynolds number and can be defined for a channel flow as $\operatorname{Re}_{\tau}=U_{\tau} h / v$, where $U_{\tau}$ denotes the friction velocity, $h$ is the channel half-height and $v$ represents the kinematic viscosity of the fluid). They stated that the mean thickness of the QC increases with an increase in the Reynolds number $\left(0.78 h\right.$ for $R e_{\tau}=1000$ compared with $0.88 h$ for $R e_{\tau}=4000$ ). Further analysis revealed that the QC has a tendency to be anti-symmetric and oscillates about the centreline of the channel.

Using direct numerical simulation (DNS) of turbulent channel flow at $\operatorname{Re}_{\tau}=930$, Yang et al. (2016) investigated the structural organization of the QC by exploring 


\section{Effect of inlet turbulence on a channel's quiescent core}

vortical structures. Utilizing the same method as Kwon et al. (2014), they found the same boundary threshold value (95\% of the centreline mean velocity) to identify the core region. They explored the population trend of the spanwise vortices in the vicinity of the core boundary, arguing that a vortex pair appears at the QC boundary consisting of a prograde and a retrograde vortex, i.e. spanwise vortices with swirling motions in the same and opposite sense as the mean shear, respectively. They found the prograde vortex to be the main provider of the mean shear near the core boundary.

Later, Jie et al. (2019) examined the influence of the QC on the dynamics of non-spherical particles in a turbulent channel flow using DNS at $R e_{\tau} \approx 1000$. Examining the rotational motion of the particles, they found that the motion of the particles also tends to be quiescent inside the QC. In their follow-up study, Jie et al. (2021) argued that it is not possible to identify a QC at $R e_{\tau}=180$. They also examined the QC impact on the spatial distribution as well as the transport of the inertial particles using DNS of a particle-laden turbulent channel flow at $R e_{\tau}=600$, showing that the particles tend to cluster inside the QC, which did exist at this $\operatorname{Re}_{\tau}$. Recent studies (Yang et al. 2019; Chen et al. 2020; Gul et al. 2020) have confirmed the presence of the core region in turbulent pipe flows as well.

The effects of incoming turbulence, i.e. freestream turbulence (FST), on a ZPG-TBL has been broadly addressed in the literature through experimental research, e.g. Blair (1983a,b), Hancock \& Bradshaw (1983, 1989), Castro (1984), Thole \& Bogard (1996), Sharp, Neuscamman \& Warhaft (2009), Dogan, Hanson \& Ganapathisubramani (2016), Dogan, Hearst \& Ganapathisubramani (2017), Dogan et al. (2019), Esteban et al. (2017), Hearst, Dogan \& Ganapathisubramani (2018), Hearst et al. (2021), Jooss et al. (2021) and, recently, using DNS, e.g. Wu, Wallace \& Hickey (2019), You \& Zaki $(2019,2020)$ and Kozul et al. (2020).

Blair $(1983 a, b)$ showed that increasing the turbulence intensity of the freestream $\left(u_{\infty}^{\prime} / U_{\infty}\right.$ up to $7 \%$, where $u_{\infty}^{\prime}$ denotes root-mean-square of the turbulent velocity fluctuations, and $U_{\infty}$ represents the mean velocity, both in the streamwise direction) increases both the skin friction and heat transfer coefficients. The author also observed that FST suppresses the wake region of the mean velocity profile, while the log region remains unaffected. Later studies also confirmed these effects of FST on a ZPG-TBL (Hancock \& Bradshaw 1983; Castro 1984; Hancock \& Bradshaw 1989; Thole \& Bogard 1996; Sharp et al. 2009; Dogan et al. 2016, 2017; Hearst et al. 2018; Dogan et al. 2019; Hearst et al. 2021; Jooss et al. 2021).

Hancock \& Bradshaw $(1983,1989)$ argued that the length scale of the FST is another parameter that affects the response of a TBL. These first studies utilized a passive grid, i.e. a fixed array of horizontal and vertical bars, to generate different FSTs, with the inherent limitation of producing relatively low turbulence intensities. This required the authors to include some measurements close to the grid (Hancock \& Bradshaw 1983; Castro 1984; Hancock \& Bradshaw 1989) to extend their parameter space, while grid turbulence is generally known to be inhomogeneous for $X / M \lesssim 20$, where $X$ and $M$ are the streamwise distance from the grid and grid mesh length, respectively (Comte-Bellot \& Corrsin 1966; Ertunç et al. 2010; Isaza, Salazar \& Warhaft 2014). The advent of the active grid, pioneered by Makita (1991), enabled turbulence researchers to generate higher turbulence intensities and control the parameters more efficiently using a single set-up. Hearst et al. (2018) utilized the ability of the active grid to produce turbulent flows with different integral length scales, while the turbulence intensity was kept approximately constant. This enabled them to investigate the effects of large-scale organizations of FST on a ZPG-TBL 43M downstream of the active grid. They concluded that the influence of the FST with higher intensities $\left(7.2 \%<u_{\infty}^{\prime} / U_{\infty}<13 \%\right)$ on the ZPG-TBL are invariant of the integral scale, at least within the range they were able to test. Similarly, Jooss et al. (2021) showed that, 


\section{Asadi, M. Kamruzzaman and R. Jason Hearst}

compared with the FST intensity, the integral scale does not play an important role in affecting the TBL for their test parameters either. It should be noted here that, unlike the external flows, for the fully developed turbulent internal flows, the size of the large-scale structures as well as the boundary layer thickness is dictated by geometry.

Employing PIV measurements in the streamwise-wall-normal plane, Dogan et al. (2019) investigated the spatial characteristics of a ZPG-TBL subjected to FST. They argued that some of the impacts of FST, such as facilitating the growth of an outer peak in the streamwise fluctuations profile, are in agreement with the effects of increased Reynolds number of canonical boundary layers and channel flows. Jooss et al. (2021) recently observed that although FST affects the wake region of a TBL, the effect diminishes with the evolution of the TBL farther downstream.

Recently, Hearst et al. (2021) analysed the instantaneous structure of a ZPG-TBL under the influence of FST using the PIV data of Dogan et al. (2019). They argued that the UMZs are present in a ZPG-TBL affected by FST; however, the increased FST levels decrease the number of the UMZs and push the upper-most UMZ edge closer to the wall.

Despite this broad range of studies on ZPG-TBL under the influence of FST, the effect of inlet turbulence on internal flows has not been investigated in great detail. One of the few exceptions is the recent work by Tuna et al. (2019), who investigated the flow development in an aspect ratio 2:1 rectangular duct with different passive grids at the inlet. They found that the shear layers met earlier for increased centreline turbulence intensities. This appears to corroborate the observations regarding the increased boundary layer thickness of ZPG-TBLs in the presence of FST (Dogan et al. 2016); however, the experiment of Tuna et al. (2019) was conducted with passive grids, limiting their producible turbulence range, and the duct itself had a low aspect ratio. The latter point is not a problem in itself, but corner effects certainly contribute to the flow evolution in a 2:1 duct (Monty 2005; Vinuesa et al. 2014), which differs from canonical expectations for a channel or pipe flow. Therefore, the present study aims to address this gap by investigating the characteristics of a turbulent channel flow under the influence of different elevated inlet turbulence levels with an emphasis on the QC. For this purpose, four different test cases were examined in a region far from the inlet of a turbulent channel flow with a matched centreline mean velocity $\left(U_{0}\right)$ but different centreline turbulence intensities $\left(u_{0}^{\prime} / U_{0}\right.$, where $u_{0}^{\prime}$ is the root-mean-square of the centreline fluctuations). It is shown that the instantaneous zonal structure of the flow is influenced by the inlet turbulence well downstream of the inlet. In $\S 2$, the experimental set-up and procedure are detailed. In $\S 3$, the effects of the inlet turbulence on the turbulence statistics are described. In $\S 4$, the core identification procedure is given. The core characteristics are detailed in $\S 5$. Finally, a summary of the findings is presented in $\S 6$.

\section{Experimental set-up and procedure}

The experiments were performed in the air channel flow facility at the Norwegian University of Science and Technology. The test section measures $50 \mathrm{~mm}$ in height $(H=$ $2 h), 600 \mathrm{~mm}$ in width $(W)$ and $5425 \mathrm{~mm}$ in length $(L)$. The aspect ratio $(W / H)$ of the channel is thus 12. This high aspect ratio ensures the two-dimensionality of the flow in the centre of the channel (Monty 2005; Vinuesa, Schlatter \& Nagib 2018). The flow is produced and manipulated using the same apparatus as described by Kamruzzaman, Rømcke \& Hearst (2021), but here a channel test section is added downstream of the inlet rather than a jet nozzle. A centrifugal fan blows air through a series of 4 screens in an expanding section. The flow then passes through a honeycomb before another screen and then a 48:1 contraction at the end of which is the test section. An active grid is placed 


\section{Effect of inlet turbulence on a channel's quiescent core}

(a)

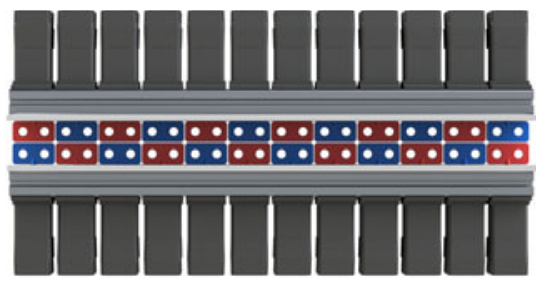

(b)

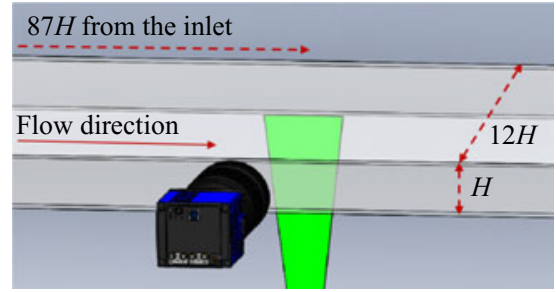

Figure 1. Schematic of $(a)$ the active grid, and $(b)$ the measurement set-up.

$2.5 \mathrm{H}$ downstream of the contraction followed by a strip of multiscale grip tape another $1 \mathrm{H}$ downstream to trip the boundary layer. Figure $1(a)$ depicts a schematic of the active grid, where the adjacent wings are shown with different colours for readability. The active grid is made up of a $12 \times 2$ array of $3 \mathrm{D}$-printed polylactic acid (PLA) plastic rectangular wings, each of which measures $49 \mathrm{~mm} \times 24 \mathrm{~mm}$ with a $3 \mathrm{~mm}$ thickness. The grid mesh length is $50 \mathrm{~mm}(1 \mathrm{H})$ and each wing is attached to its own stepper motor (Moons' STM17S-3RE). Prescribed random signals of rotational direction, velocity, and duration were sent to each motor. The walls of the channel were built out of acrylic sheets to provide optical access.

Two-dimensional, two-component PIV measurements in the streamwise-wall-normal plane were performed $87 \mathrm{H}$ downstream of the inlet in the centre of the channel. The schematic of the PIV measurement set-up is illustrated in figure 1(b). A 16-bit LaVision Imager sCMOS camera $(2560 \times 2160$ pixels $)$ equipped with a Zeiss Milvus ${ }^{\circledR} 2 / 100 \mathrm{M}$ lens was aligned to cover an approximately $50 \mathrm{~mm}(H) \times 44 \mathrm{~mm}(0.88 H)$ area of the flow field in height and length, respectively. Consequently, each pixel covered an area of approximately $20 \mu \mathrm{m} \times 20 \mu \mathrm{m}$, resulting in a magnification factor of 0.325 . The field of view was illuminated by a dual-pulse Nd:YAG laser (Litron Nano L 200-15 PIV with $200 \mathrm{~mJ}$ per pulse). The laser beam was turned into a sheet and focused over the field of view by passing it through a pair of spherical lenses followed by a cylindrical lens (LaVision light sheet optics). Tracer particles with a mean diameter of approximately $1 \mu \mathrm{m}$ were produced using a Martin Magnum $2500 \mathrm{~Hz}$ smoke generator and fed to the flow at the entrance of the fan. Owing to the high sensitivity of the camera and adequate illumination power, the lens $f \#$ was set to 11 , which compensated for the small size of the particles and yielded a particle size of approximately 2-3 pixels (see Smith \& Neal 2016; Raffel et al. 2018). Examining the histograms of sub-pixel displacements revealed that this helped to avoid pixel-locking issues (e.g. there is no spurious peaks in p.d.f.s of the modal velocity presented later in $\$ 4.1$ ). For each test case, 5100 independent image pairs were acquired at $15 \mathrm{~Hz}$ to provide sufficient vector fields for the targeted analyses. This also ensures the convergence of first- and second-order statistics. LaVision DaVis 10.1 was used to record and process the image pairs. An iterative cross-correlation process was performed using a 96 pixels $\times 96$ pixels window size with an overlap of $50 \%$ as the first pass, with a 48 pixels $\times 48$ pixels window with a $75 \%$ overlap for the final pass. The estimated uncertainties by DaVis, which are estimated by established correlation methods (Sciacchitano et al. 2015; Wieneke 2015), were approximately $3 \%$ of the centreline mean velocity in the near-wall region and decreased to $\approx 1 \%$ away from the walls. Measurements were also performed at $100 \mathrm{H}$ downstream of the inlet acquiring fewer (2000) image pairs. The results generally agree with that of the main measurements and are detailed in Appendix A.

A Scanivalve MPS4264 miniature pressure scanner with a $\pm 4 \mathrm{inH}_{2} \mathrm{O}$ full-scale range and $\mathrm{a} \pm 2 \%$ accuracy of the full scale was utilized to measure the pressure drop along the 


$\begin{array}{lccc}\text { Case } & U_{\tau}\left(\mathrm{m} \mathrm{s}^{-1}\right) & \Omega \pm \omega(\mathrm{Hz}) & u_{0}^{\prime} / U_{0}(\%) \\ \text { REF } & 0.460 & \text { Static } & 3.7 \\ \text { A } & 0.470 & 9 \pm 3 & 4.8 \\ \text { B } & 0.468 & 4.5 \pm 1.5 & 4.9 \\ \text { C } & 0.466 & 0.75 \pm 0.25 & 6.4\end{array}$

Table 1. Active grid and flow parameters of the test cases.

channel at 16 pressure ports. The taps were spaced in intervals of $325 \mathrm{~mm}(6.5 \mathrm{H})$ with the first one located $7.5 \mathrm{H}$ downstream of the inlet. The relative pressure drop was measured for all ports simultaneously at $800 \mathrm{~Hz}$, while the first port was used as the reference. Measurements were conducted for 10 minutes to ensure convergence of the time-varying signals. The wall shear stress $(\tau)$, and resulting friction velocity $\left(U_{\tau}\right)$, were estimated by fitting a line to the mean pressure drop data for pressure taps $8-15$. The linearity of the measured pressure gradient resulted in coefficient of determination $\left(R^{2}\right)$ values generally greater than 0.995 .

Four different test cases were investigated, i.e. the reference case REF where the active grid was left in its fully open position, acting as a flow straightener, together with three different active grid sequences (active cases) which are referred to as cases A, B and C, with increasing centreline turbulence intensity $\left(u_{0}^{\prime} / U_{0}\right)$. In all three active cases, the wings were actuated using fully random sequences as described by Hearst \& Lavoie (2015). The actuator signals were random with a $\Omega \pm \omega$ top-hat distribution, where $\Omega$ is the mean rotational velocity and $\omega=\Omega / 3$ is the limiting parameter. The centreline mean velocity $\left(U_{0}\right)$ was kept constant at $10 \mathrm{~m} \mathrm{~s}^{-1}$ within $1 \%$ for all cases, resulting in a matched centreline Reynolds number of $\operatorname{Re}_{H}=33200$. Further details regarding the test cases are given in table 1.

Although previous studies have shown an increase in the friction velocity of a ZPG-TBL under the influence of FST (Blair 1983a; Hancock \& Bradshaw 1983; Sharp et al. 2009; Dogan et al. 2016; Esteban et al. 2017), the friction velocity was observed to be constant within $2 \%$ for the different cases tested here. This resulted in an approximately matched friction Reynolds number of $R e_{\tau} \approx 770$. This suggests that the inlet turbulence intensity has no effect on $U_{\tau}$ of the turbulent channel flow for different cases tested in the current study. The spatial resolution of the PIV measurements was approximately 30 wall units $\left(y^{+}\right)$, which was deemed to be acceptable considering that the focus of the core analysis is the outer regions of the velocity profile. Nevertheless, three rows of the processed vector fields in the vicinity of the top and bottom walls were discarded to prevent unreliable observations in the near-wall region. Another noteworthy point is the increased centreline turbulence intensity with decreased actuation velocities $(\Omega)$ of the grid. This is consistent with trends reported by previous active grid studies in wind tunnels (Hearst \& Lavoie 2015; Dogan et al. 2016; Hearst et al. 2018).

Modifying the post-processing method originally developed by Rodríguez-López, Bruce \& Buxton (2015), average velocity profiles were compared with the canonical description of the channel flow to correct the location of the upper and lower wall. To do this, a set of parameters, including the von Kármán constant, were obtained by minimizing the residual error with respect to the description of the canonical channel flow, namely, modified Musker profile and the log law, to which a channel flow wake function of Nagib \& Chauhan (2008) was added. 

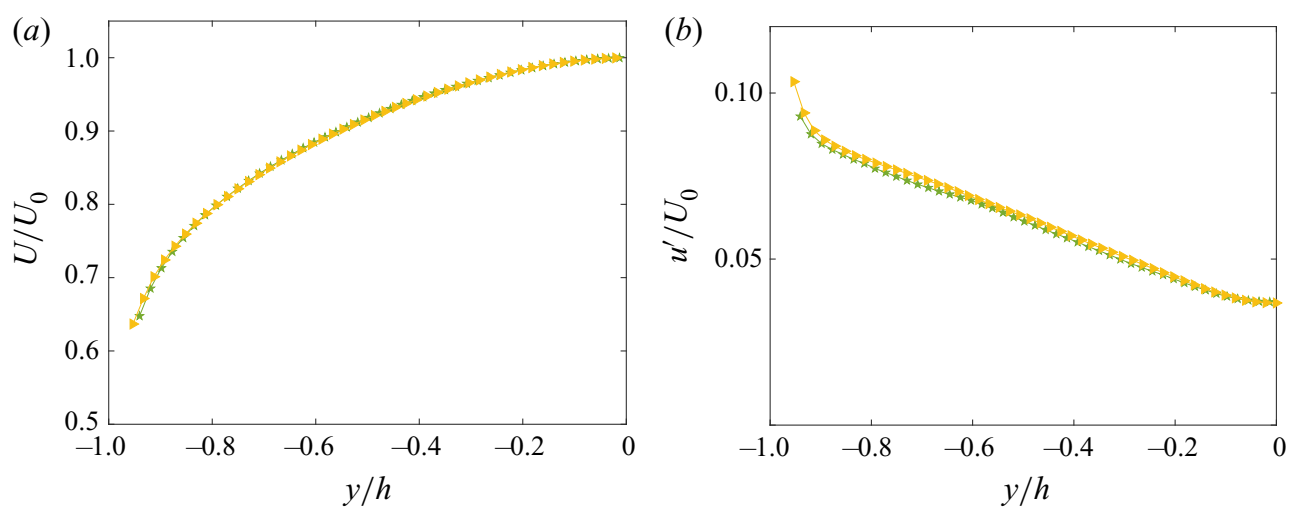

Figure 2. (a) Mean velocity, and (b) streamwise turbulence intensity profiles normalized by the centreline mean velocity for REF at $X / H=87$ ( $\downarrow$, yellow), and $X / H=100$ ( $\star$, green).

\section{First- and second-order flow statistics}

In order to assess the streamwise evolution of the flow, an auxiliary PIV campaign was carried out $100 H$ downstream of the inlet, recording 2000 image pairs for each test case. The first- and second-order statistics of the REF case at two streamwise locations $(X / H=$ 87 and 100, where $X$ denotes a streamwise coordinate whose origin is at the channel inlet) are illustrated in figure 2, and are representative of all cases. Figure 2(a) compares the mean velocities in the same manner as Schultz \& Flack (2013), while figure 2(b) shows the turbulence intensity profiles. For quantification, the velocity and turbulence intensity data at two different streamwise locations were interpolated on a common grid. The resulting maximum root-mean-square deviation for different test cases was in a range of $0.24 \%-0.43 \%$ and $0.16 \%-0.36 \%$, for mean velocities and turbulence intensities, respectively. Bearing the uncertainty of the measurements in mind, the deviations are small enough to neglect the streamwise evolution of these statistics at the first measurement station where a greater number of images were collected.

Figure 3(a) shows inner-normalized mean streamwise velocity profiles together with DNS data of a turbulent channel flow at $R e_{\tau} \approx 1000$ with a size of $L=8 \pi h, H=2 h$, and $W=3 \pi h$ (Graham et al. 2016). It is shown that the added turbulence does not affect the mean velocity profile of the channel in the log layer significantly, similar to the previous observations for a ZPG-TBL under the influence of FST (Blair 1983a; Hancock \& Bradshaw 1983, 1989; Thole \& Bogard 1996; Sharp et al. 2009; Dogan et al. 2016; Hearst et al. 2018; Dogan et al. 2019; Jooss et al. 2021). On the other hand, in the outer layer, the inlet turbulence suppresses the wake region. In order to quantify this effect, the Coles' wake parameter $(\Pi)$ is calculated for the different cases fitting the description of canonical channel flow to the data as mentioned in the previous section. The obtained wake parameters are $0.076,-0.006,-0.007$ and -0.020 for the cases REF, A, B and $\mathrm{C}$, respectively, indicating a suppressive impact on the wake region by the increased centreline turbulence intensity. Again, this effect was previously reported for a ZPG-TBL subjected to FST (Blair 1983a; Hancock \& Bradshaw 1983; Thole \& Bogard 1996; Sharp et al. 2009; Dogan et al. 2016; Jooss et al. 2021). However, the wake suppression effect is more prominent in a ZPG-TBL under the influence of FST because the wake region is much stronger in a ZPG-TBL than that of a turbulent channel flow (Jiménez et al. 2010).

Figure 3(b) shows inner-normalized streamwise and wall-normal turbulent fluctuations, as well as Reynolds shear stress. The streamwise turbulent fluctuations are primarily 
(a)

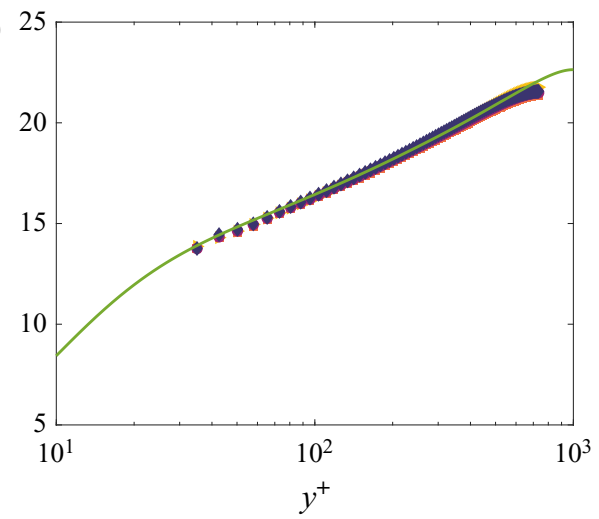

(b)

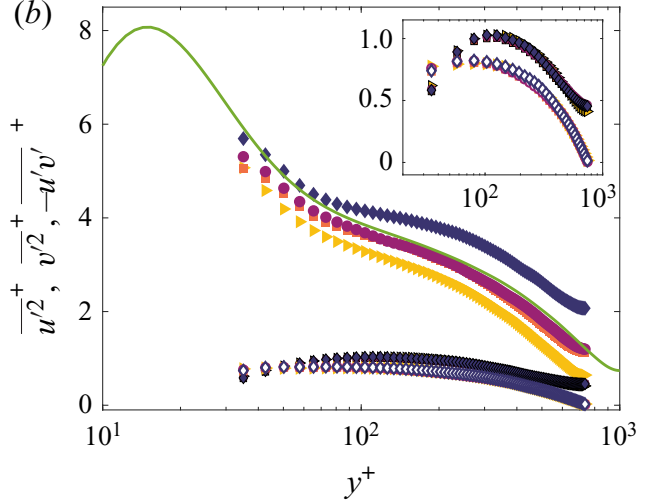

Figure 3. Inner-normalized profiles of ( $a$ ) mean velocity and $(b)$ streamwise (filled symbols) and wall-normal (symbols with black border) turbulent fluctuations, as well as Reynolds shear stress (open symbols) for cases REF ( $\bullet$, yellow), A ( $\square$, orange), B (•, purple) and C ( $\downarrow$, navy blue). The inset is zoomed in on the wall-normal fluctuations and Reynolds shear stress profiles, and the data sets are downsampled for clarification. The solid green line indicates statistics of DNS data at $R e_{\tau} \approx 1000$ (Graham et al. 2016).

different near the channel centreline and appear to approach each other near the walls. This is markedly different from observations in ZPG-TBLs where the near-wall streamwise variance is also considerably different between cases (Sharp et al. 2009; Dogan et al. 2016; Hearst et al. 2018; Dogan et al. 2019; Jooss et al. 2021). In contrast, the wall-normal turbulence fluctuations and the Reynolds shear stress do not have substantial differences between the cases. The fact that the Reynolds shear stress is not affected by the inlet turbulence intensity for the internal flow may be the reason for an approximately constant $U_{\tau}$ across the cases investigated herein, while the similar cases in a ZPG-TBL influenced by FST would result in different Reynolds shear stress profiles and $U_{\tau}$ (see Dogan $e t$ al. (2019), figure 5). Thus, the effects of varying the incoming turbulence intensity are primarily on the streamwise fluctuations and predominantly located about the centre of the channel.

\section{Core identification}

The peaks of the instantaneous velocity histogram correspond to modal velocities, which represent the driving velocity of a UMZ (Adrian et al. 2000). The so-called QC of turbulent channel flow is the UMZ associated with the largest peak of the instantaneous velocity. Kwon et al. (2014) identified the local maxima in the p.d.f. of instantaneous velocity fields, showing that the number of peaks is a function of the streamwise domain. Nonetheless, they stated that the two highest peaks are preserved in the histogram of the streamwise velocity, increasing the streamwise domain up to $6 h$ (see their figure $3 d$ ). Thus, they represent the two most robust uniform momentum zones of the flow. Seeking a more reliable method, they repeated this procedure for all PIV fields and plotted the p.d.f. of all identified modal velocities. They defined a constant threshold for the QC boundary as the value of the first local minimum $\left(0.95 U_{0}\right)$ below the dominant peak. Subsequently, the same method was adopted by Yang et al. (2016) and Jie et al. $(2019,2021)$ to identify the threshold value of the QC in their turbulent channel flow, yielding the same constant value of $0.95 U_{0}$. Application of these techniques to the present data is described in $\S 4.1$ and a further extension of the methodology is given in $\S 4.2$. 


\section{Effect of inlet turbulence on a channel's quiescent core}
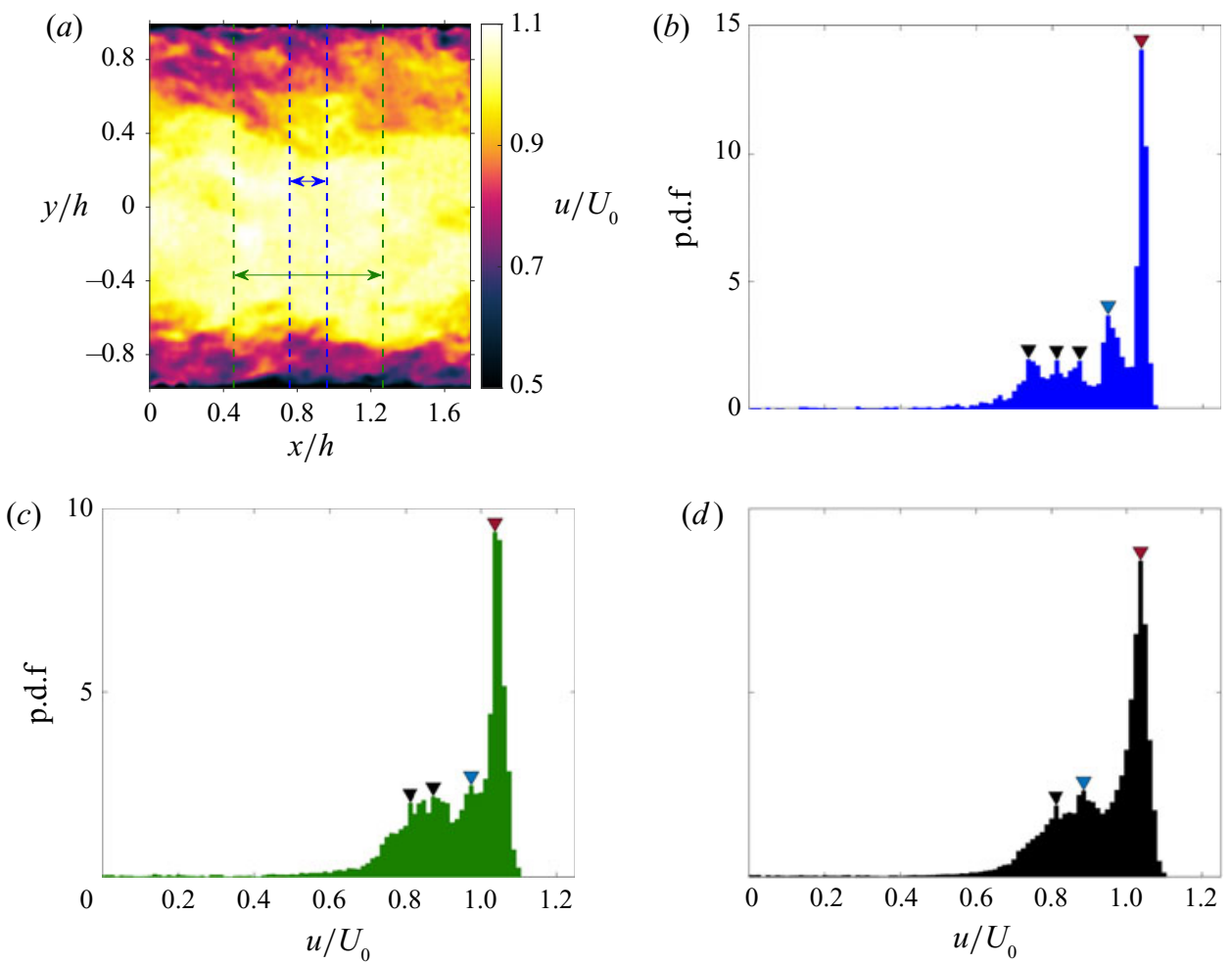

Figure 4. An example of peak detection procedure. (a) An instantaneous streamwise velocity field of case C. Blue dashed lines demarcate the initial subregion in the peak detection procedure $(\delta x \simeq 0.2 h)$, while green dashed lines mark the boundaries of the mid (5th) subregion $(\delta x \simeq 0.8 h)$. Histograms of the instantaneous streamwise velocity for $(b)$ the initial subregion, $(c)$ the mid subregion, and $(d)$ the final subregion $(\delta x \simeq 1.7 h)$. Detected peaks are illustrated with down triangles. Crimson and blue down triangles mark the highest and the second highest peak, respectively.

\subsection{Identification of the $Q C$}

Starting with a streamwise domain length of $0.2 h$, the peaks are identified in the histograms of instantaneous PIV fields. Varying the streamwise extent from $0.2 h$ to $1.7 h$ in 10 steps (found to be sufficient to converge the modal velocity p.d.f.s), the modal velocities are identified for the same PIV field with different streamwise domain lengths. Figure 4 shows an example of this procedure for a sample velocity field as well as three histograms of instantaneous streamwise velocity for three different domain extents. The detected peaks are marked with down triangles. Repeating this procedure for all PIV fields yields the p.d.f. of all detected modal velocities, plotted in figures $5(a-d)$. As shown in figure $5(a)$, the p.d.f. of the REF case has a dominant peak at $u=U_{0}$. Moving towards the lower velocities, it drops until a minor minimum is exposed. On the other hand, the distribution of the modal peaks is considerably altered for the active cases (figures $5(b-d)$ ). The added turbulence increases the streamwise velocity fluctuations, hence altering the distribution of the modal velocities. This, in turn, causes difficulties for defining the QC boundary threshold value based solely on these p.d.f.s.

Accordingly, the above-mentioned procedure is modified here to identify the threshold value of the QC boundary in these flows with more extreme turbulence. Starting with a streamwise domain length of $0.2 h$, only the first and second highest peaks, e.g. the red 


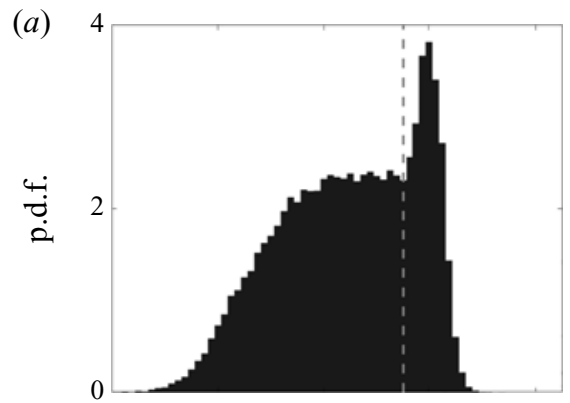

(e) 15
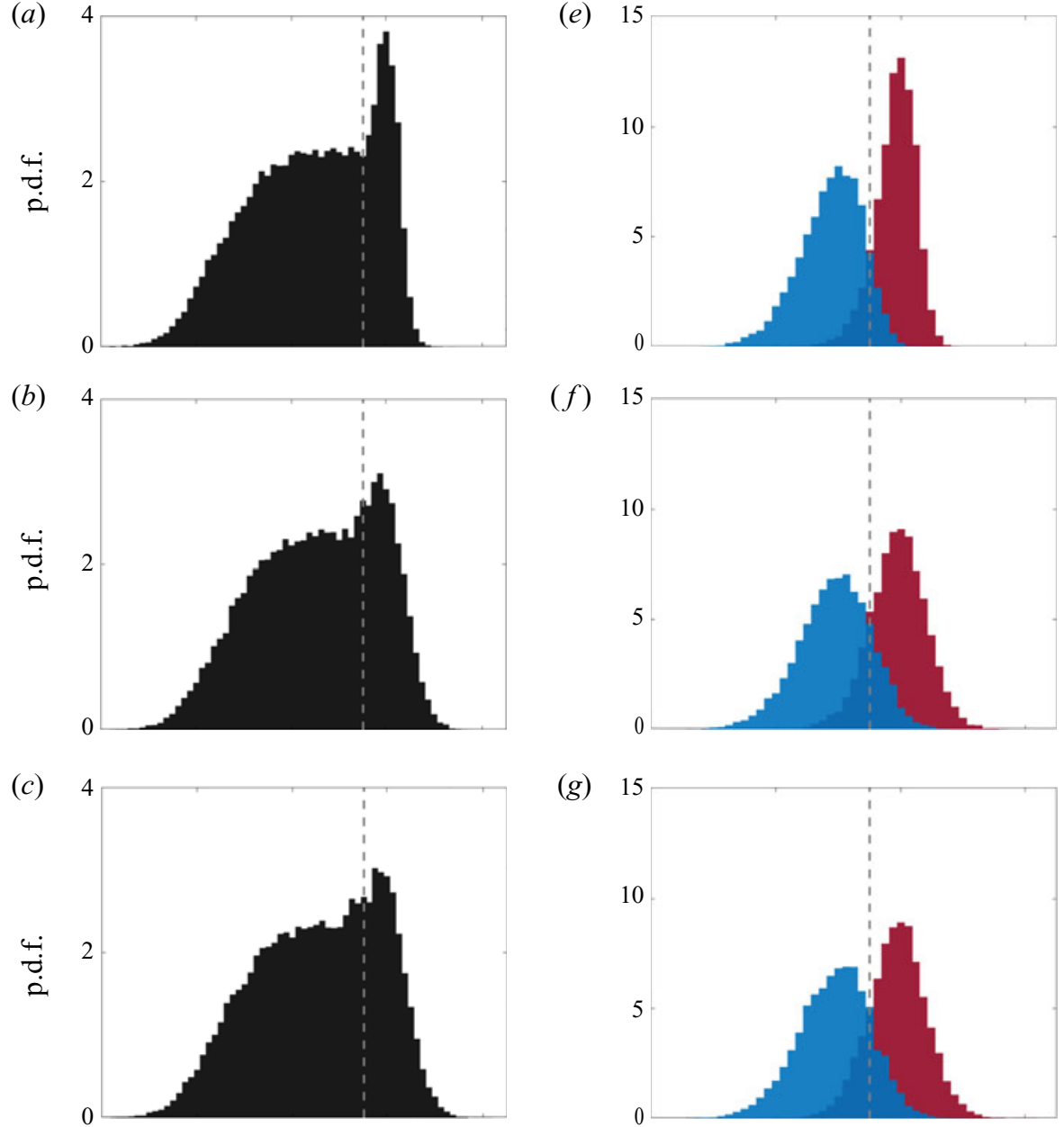

(g)
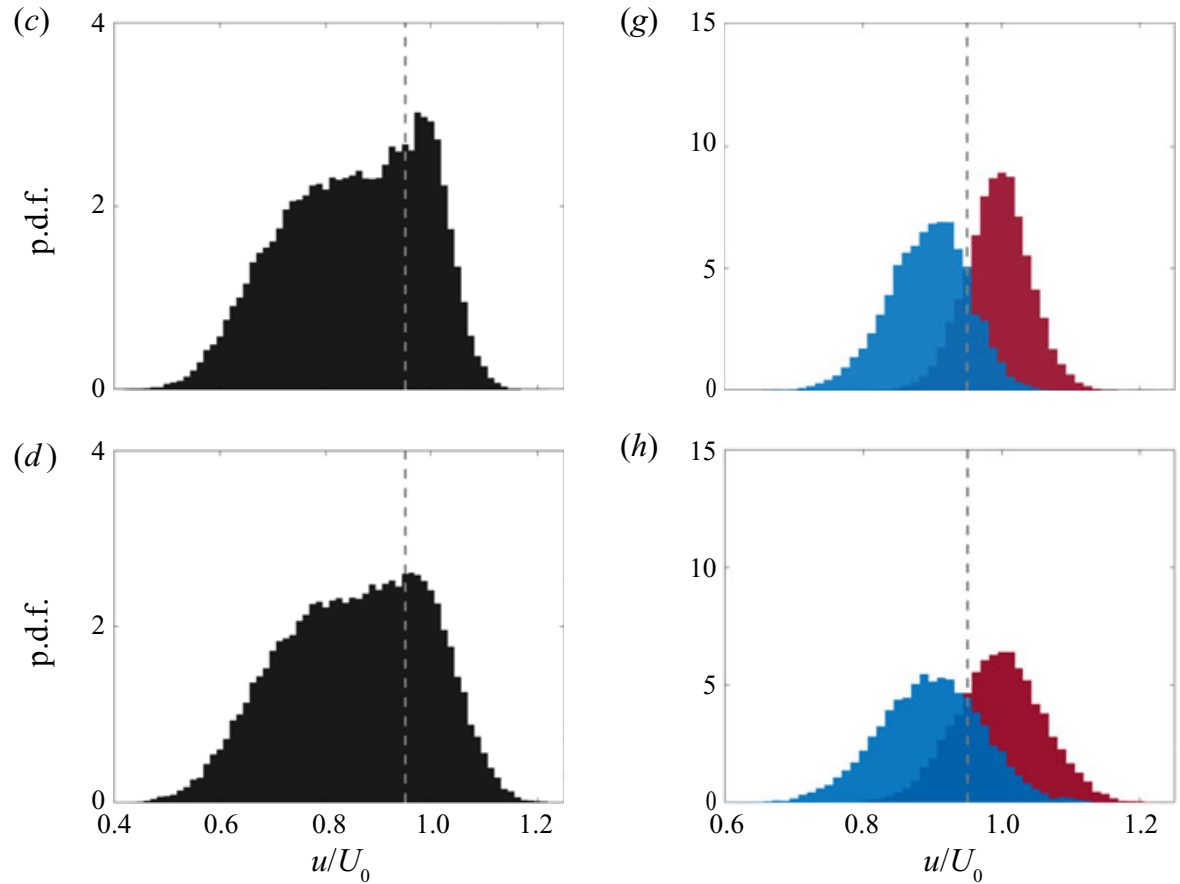

Figure 5. p.d.f.s of $(a-d)$ all the modal velocities, and $(e-f)$ the highest (crimson) and the second highest (blue) modal velocity, found in all PIV fields for cases REF, A, B and C, respectively, from top to bottom. The grey dashed lines mark $u=0.95 U_{0}$. 


\section{Effect of inlet turbulence on a channel's quiescent core}

and blue down triangles in figures $4(b-d)$ are identified in the histograms of instantaneous PIV fields as the representatives of the two most robust UMZs. Similarly, varying the streamwise subdomain extent from $0.2 h$ to $1.7 h$ in 10 steps, the two modal velocities are identified for the same PIV field with different streamwise domain lengths, and this procedure is repeated for all PIV fields. Figures $5(e-h)$ show the distribution of the first and the second modal velocity separately. As illustrated in figure 5(e), a distinct valley is exposed at $u=0.95 U_{0}$ between the p.d.f.s of the first and the second modal velocity. In a similar and less ambiguous manner, this can be defined as the threshold value of the core boundary.

The prominence of the peaks and troughs diminishes due to the spread of the p.d.f.s under the influence of the added turbulence (figures $5(f-h)$ ). This is most pronounced for the most turbulent case (figure $5 h$ ), where the distribution of the modal velocities is flatter around the peak. Considering the methodology described above, $u_{t h}=0.95 U_{0}$ is chosen for all the test cases as the QC identification threshold.

Although the highest peak is always detectable in the histograms of the instantaneous velocity, the second highest peak cannot be identified in $13.1 \%, 16.7 \%, 16.1 \%$ and $19.7 \%$ of the total histograms for REF, A, B and C, respectively. Nevertheless, in the majority of the velocity fields, the two highest peaks were identifiable, which means that the results presented herein represent the majority of the flow events passing through the channel. In addition, some other crucial factors affect the identification of instantaneous modal velocities. One of which is the streamwise domain length addressed earlier. Other factors include bin size as well as threshold parameters used for detecting the peaks, i.e. peak prominence, minimum peak distance and peak height. Sensitivity analysis was performed, indicating that the location of the troughs (presented in figure $5(e-h)$ ) is fairly robust to the variation of these parameters. Further, severe variations of the mentioned parameters shift the threshold value by approximately $\pm 0.01 U_{0}$, which does not change any of the trends presented in this study. An alternative approach would be to use kernel density estimation of the histogram as proposed by Fan et al. (2019); however, this approach generally identifies fewer peaks (Chen et al. 2020; Chen, Chung \& Wan 2021), which in turn hinders the method employed in this study to identify the core boundary threshold value, especially given that typically a distinct region of the flow with a uniform streamwise velocity (core) is present in the instantaneous fields. The procedure described above adjusts the original method of Kwon et al. (2014) to detect the boundary threshold value of the QC with less ambiguity.

Figure 6 illustrates three different instantaneous velocity fields of case C. Employing the identified threshold value, the QC can be defined as the region bounded by the long continuous contour lines of $u_{t h}=0.95 U_{0}$. Similar to the approach of Kwon et al. (2014), the small closed contours identifying a limited region of similar velocity (illustrated with thin blue lines in figure 6) are neglected throughout this study. A classic continuous QC is illustrated in figure 6(a). The QC region is demarcated by two continuous contour lines of $0.95 U_{0}$. This kind of core is present in the majority of the instantaneous velocity fields. However, in some of the PIV snapshots, as demonstrated in figure $6(b)$, the core is discontinuous. In other words, the boundary lines do not span the whole streamwise extent of the PIV domain; instead, one of them begins and ends on the upstream edge of the domain, while the other one is located at the downstream edge. These two types of cores have been observed previously by Kwon et al. (2014).

More interestingly, figure 6(c) shows an instantaneous velocity field in which the core is unidentifiable using the threshold value of $u_{t h}=0.95 U_{0}$. These instances are observed only for the active cases (cases A, B and C), in which there is no QC boundary as defined for the ordinary and discontinuous QCs, though some small contour lines of $u=0.95 U_{0}$ 


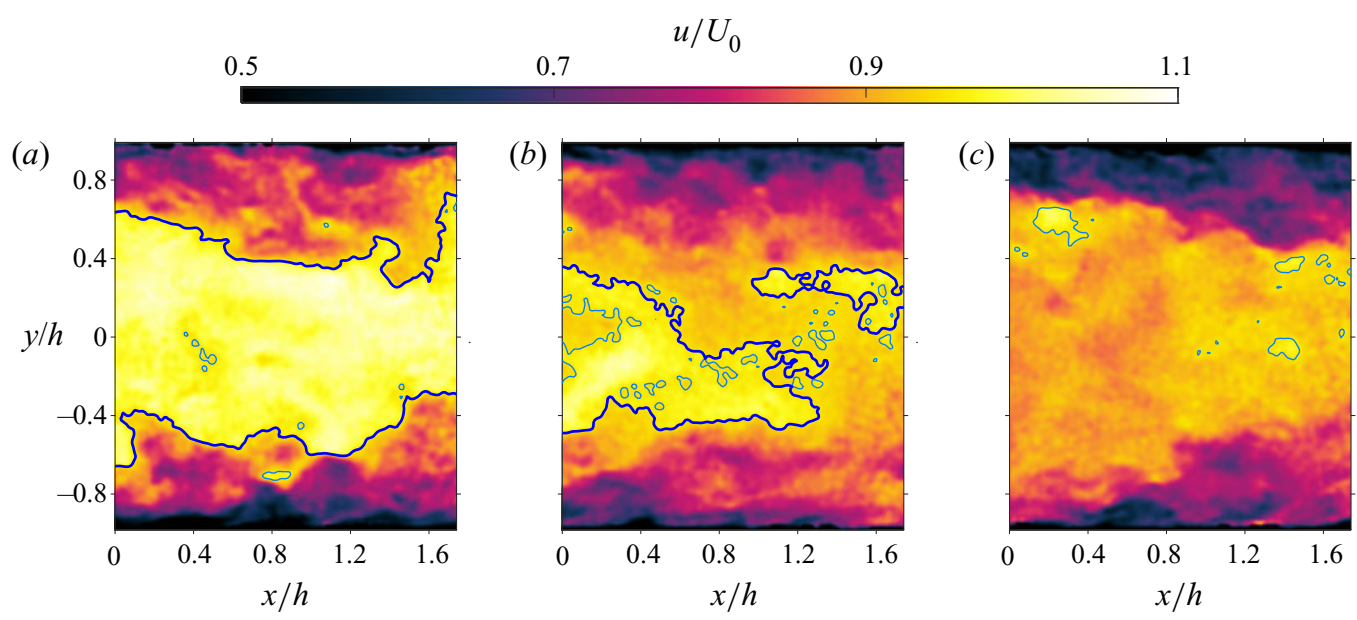

Figure 6. Sample instantaneous streamwise velocity fields for case C, showing $(a)$ a continuous core, $(b)$ a discontinuous core and $(c)$ a 'No QC' example. Contour lines of $u_{t h}=0.95 U_{0}$ are indicated with blue lines. The thicker lines highlight the QC boundary.

$\begin{array}{lccc}\text { Case } & \text { Continuous } & \begin{array}{c}\text { No. of cores }(\%) \\ \text { Discontinuous }\end{array} & \text { No QC } \\ \text { REF } & 97.0 & 3.0 & 0 \\ \text { A } & 89.8 & 7.9 & 2.3 \\ \text { B } & 89.0 & 8.4 & 2.6 \\ \text { C } & 79.6 & 9.4 & 11\end{array}$

Table 2. Presence of the different cores in the velocity fields of each test case as a percentage of the total fields (5100).

are observed. In effect, the QC of the channel flow is not present across the PIV domain. These instances are referred to here as 'No QC' cases. The identification of the core region in these instances is explored further in $\S 4.2$. It should be noted that all types of the QC mentioned above might be dependent on the streamwise extent of the PIV domain, e.g. a discontinuous core may be observed as a continuous core in a smaller domain or vice versa.

Table 2 lists the percentage of the velocity fields with the different types of the QC. It is apparent that the 'No QC' instances are present only for the active cases, and their number increases with turbulence intensity. This is also in agreement with the trends observed in figure 5, where the increased levels of the turbulence intensity mitigated the prominence of the trough used to define the boundary threshold value of the QC. The discontinuity of the cores is also found to increase with turbulence intensity. However, the growth rate of the 'No QC' instances appears to be higher than that of the discontinuous cores.

Figure 7 illustrates the vertical gradient field of the streamwise velocity for figure $6(a)$. As shown, the QC boundary passes through a series of high gradient regions, which is typical of a UMZ boundary and indicates a sudden change in the streamwise velocity as the boundary of the QC is crossed in the vertical direction (Kwon et al. 2014; Eisma et al. 2015; de Silva et al. 2016; Hearst et al. 2021). 


\section{Effect of inlet turbulence on a channel's quiescent core}

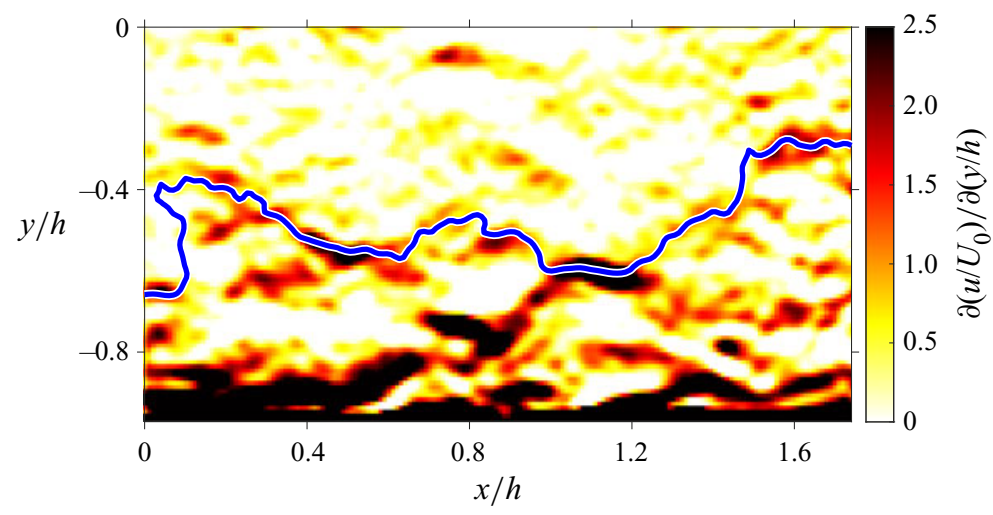

Figure 7. The instantaneous field of the streamwise velocity gradient for figure $6(a)$ in the lower half of the channel. The blue line indicates the core boundary.

\subsection{Identification of new core states}

As shown in $\S 4.1$, the QC is not present in a number of PIV snapshots for the active cases. A question that arises here is if an alternative repeating structure is identifiable in these velocity fields. In search of such structure, the 'No QC' instances are re-examined employing the same p.d.f. analysis used for identification of the QCs. The resulting p.d.f. is shown in figure 8(a) for the most turbulent case (case C). Similar to figure 5, a valley is evident between the p.d.f.s of the first and the second modal velocity. However, it is clear that the exposed valley, as well as the peaks, are more prominent than that of figure $5(h)$. The valley corresponds to $u / U_{0} \approx 0.85$. Consequently, opting for this new threshold value to identify the core region of these velocity fields, the actual instantaneous core region of the channel flow is identified. Figure $8(b)$ illustrates the new core region discovered for figure $6(c)$ using the updated boundary threshold value. A close look at figure $8(a)$ reveals that the p.d.f. of the first peak moves to lower velocities, indicating that generally 'No QC' instances correspond to negative fluctuations of the streamwise velocity in the central regions of the channel. Consequently, these newly identified cores bear a bulk of fluid with lower momentum than that for the conventional QCs. This further explains why it was not possible to find the core boundaries using a constant threshold value of $u_{t h}=0.95 U_{0}$. The same procedure is repeated for the other active cases. For these test cases (case A and B), the threshold value was found to be $0.87 U_{0}$.

At this point, one may wonder there are high-momentum counterparts for the newly identified low-momentum cores. These high-momentum cores would correlate with positive fluctuations of the streamwise velocity. The lower threshold for the low-momentum cores suggest that the actual boundary of a high-momentum core should correspond to a value greater than the global threshold $\left(u_{t h}=0.95 U_{0}\right)$. Nonetheless, these cores are not readily evident since they would be contained within the boundaries of the traditional QCs identified with the $0.95 U_{0}$ threshold. In order to examine this conjecture, a criterion should be defined to identify these fields and examine them.

Accordingly, a presumed threshold value higher than the universal one is assumed for this new core state. Afterward, the velocity fields in which the contour lines of this presumed threshold form continuous boundaries across the domain are picked. Finally, analysing the modal velocity p.d.f.s of these fields should reveal the actual value of the core boundary threshold. 

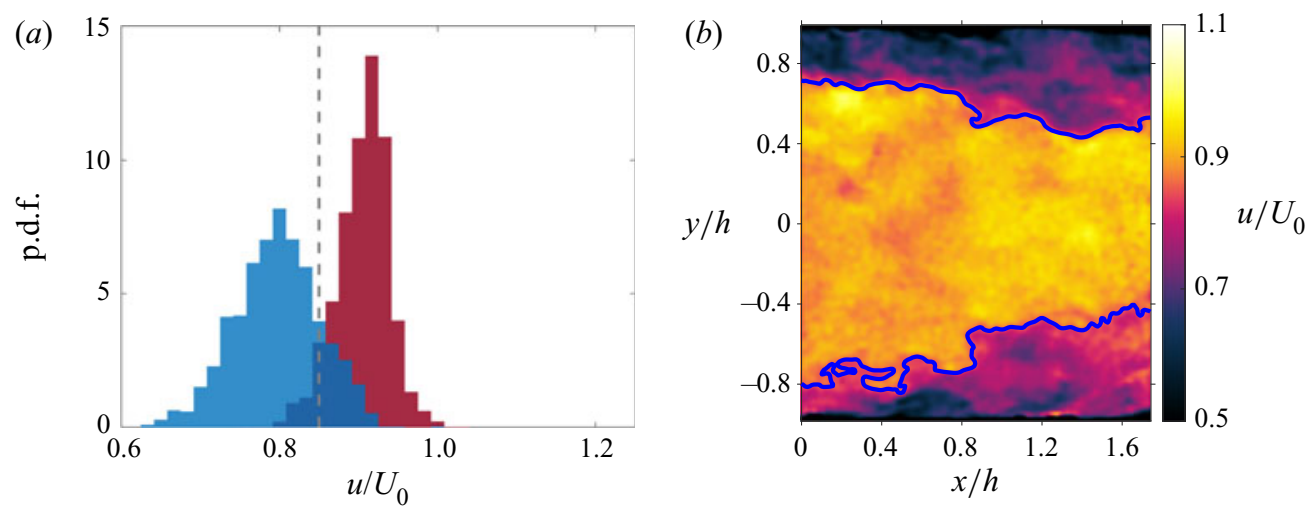

Figure 8. (a) p.d.f.s of the first (crimson), and the second (blue) modal velocity found for 'No QC' fields of case $\mathrm{C}$ with varying the streamwise domain from $0.2 h$ to $1.7 h$. The grey dashed line marks $u=0.85 U_{0}$. $(b)$ The same field as illustrated in figure $6(c)$ with contour lines of $u_{t h}=0.85 U_{0}$ delineating the new (low-momentum) core boundaries.

The presumed threshold value was chosen to be $1.065 U_{0}$ so that the number of the newly identified fields is approximately the same as the 'No QC' instances that have now been attributed to low-momentum cores. Figure $9(a)$ illustrates the p.d.f.s of the modal velocities for the newly identified fields of case $\mathrm{C}$. In this figure, a trough is present at $\approx 1.04 U_{0}$, which is selected as the core boundary threshold for these velocity fields. Figure $9(b)$ shows an instance of these fields for case C. Looking closely at this figure reveals that the inner-most UMZ is bounded by the boundary lines of $u_{t h}=1.04 U_{0}$ (thick blue lines). This region is considered as the actual core of the channel in this instantaneous velocity field, even though an area is bounded by the boundary lines of $u=0.95 U_{0}$ (thin blue lines). As expected, these new core regions contain a bulk of high-momentum fluid compared with the conventional QCs as the p.d.f. of the highest modal velocity is shifted to higher velocities. Repeating the same analysis for the less turbulent cases A and B yielded a threshold value of $1.03 U_{0}$, slightly lower than that for the most turbulent case. A sensitivity analysis is detailed in Appendix B to assess the effects of changing the presumed threshold value on the results.

As illustrated in this section, inlet turbulence breaks down the universality of the QC boundary threshold, i.e. it is not possible to identify the actual core of the channel flow using a constant threshold value for all the velocity fields. In addition, for increased turbulence intensities, the characteristic velocity of the core significantly deviates from the centreline mean velocity in some instances. New threshold values were found for these specific velocity fields with strong negative and positive fluctuations of the streamwise velocity inside the inner-most UMZ, which in turn led to the identification of new core states with different momentum levels. These new core states have been referred to as low momentum and high momentum to identify the cores that occur as a result of a bulk of low-momentum or high-momentum fluid, respectively, compared with the conventional definition of a QC; hereafter, we will refer to them as 'low-momentum core' (LMC), 'ordinary core' (OC) and 'high-momentum core' (HMC), each of which has a local boundary threshold value. Table 3 lists the number of the identified cores as the percentage of the total PIV fields for each case. As expected, the occurrence of both LMC and HMC increases with an increase in the centreline turbulence intensity. Furthermore, these new core states are not present in the velocity fields of REF. 

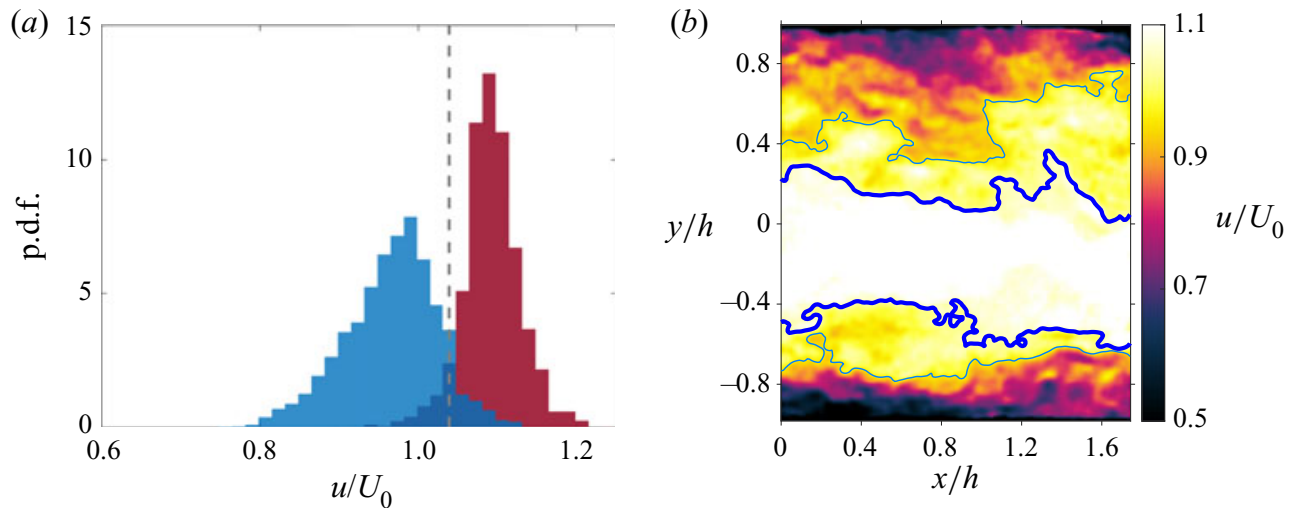

Figure 9. (a) p.d.f.s of the first (crimson), and the second (blue) modal velocity found for high-momentum fields of case $\mathrm{C}$ with varying the streamwise domain from $0.2 \mathrm{~h}$ to $1.7 \mathrm{~h}$. The grey dashed line marks $u=1.04 U_{0}$. (b) An instantaneous streamwise velocity field of case $\mathrm{C}$ containing a bulk of high-momentum flow within the standard QC. Thin blue lines indicate the standard QC boundaries (contour lines of $u_{t h}=0.95 U_{0}$ ), while the two thicker blue lines demarcate the new high-momentum core identified by a threshold of $u_{t h}=1.04 U_{0}$.

\begin{tabular}{lccc} 
Case & \multicolumn{3}{c}{ No. of continuous core states (\%) } \\
& LMC & OC & HMC \\
REF & 0 & 97.0 & 0 \\
A & 2.3 & 86.6 & 3.2 \\
B & 2.6 & 85.8 & 3.4 \\
C & 11.0 & 68.2 & 11.4
\end{tabular}

Table 3. Presence of the various states of the continuous core, identified by the local threshold values, in the velocity fields of each test case as a percentage of the total fields (5100). Note, the remaining percentage of cores are discontinuous.

As mentioned previously, a sharp change in the streamwise velocity occurs at the boundary of a UMZ. This was also shown previously in figure 7 where the core boundary passed through high-gradient regions as an indicator of a sharp change in the streamwise velocity. Figure 10, in the same manner, depicts the vertical gradient fields of the streamwise velocity for the PIV snapshots shown in figures $8(b)$ and $9(b)$. There is an evident co-location between the edges of the new core regions and high-shear zones. Moreover, as illustrated in figure $10(b)$, the boundary line of the new core region (thick blue line) passes through separate high-shear zones compared with the previous boundary (thin blue line), identifying that this is in fact a UMZ and that there are significant shear events above the $0.95 U_{0}$ threshold, i.e. that threshold does not identify a 'quiescent' region of the flow in this particular case. This thus affirms the validity of the new core boundary.

\section{Statistical analysis of the cores' structure}

It has already been found that the inlet turbulence manipulates the characteristic velocity of the cores, hence their momentum. In order to further investigate the effects of the inlet turbulence on the channel core, several characteristics are analysed in this section. As a prerequisite for some of the analyses, the core boundaries need to be single-valued functions of the streamwise coordinate. Due to the folding of the boundary lines, this 


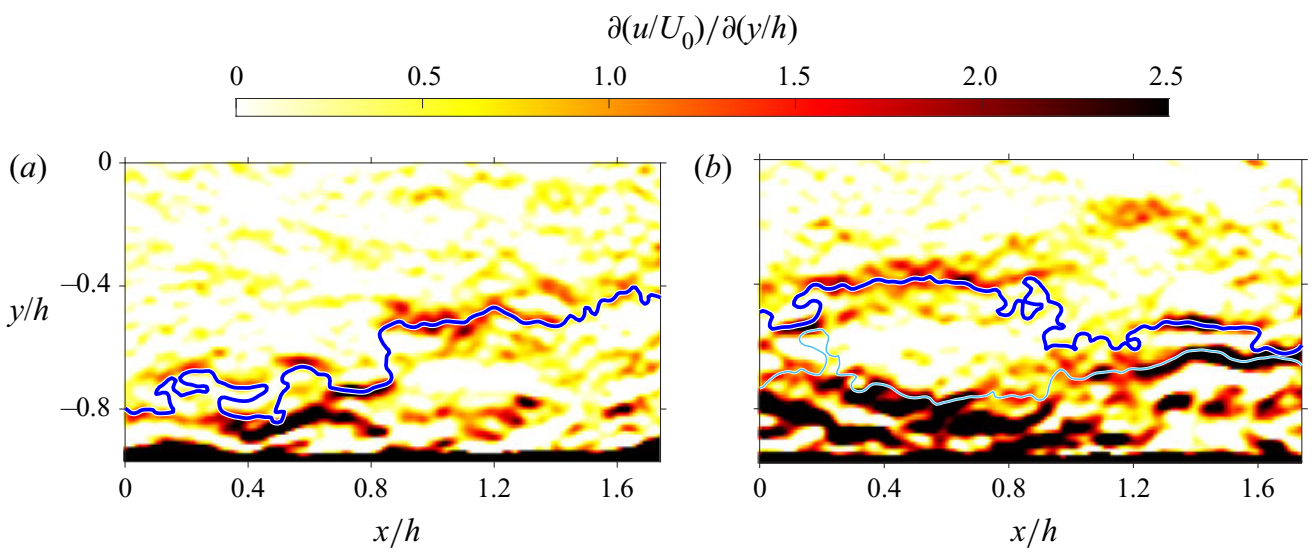

Figure 10. Instantaneous fields of streamwise velocity gradient in the lower half of the channel for the same field as $(a)$ figure $8(b)$, and $(b)$ figure $9(b)$. The thick blue line identifies the new core boundary. The thin blue line indicates the previously identified core boundary using $u_{t h}=0.95 U_{0}$.

is not always the case. As a remedy, Kwon et al. (2014) used the inner-enveloped core boundary method, which turns the core contour into a function by always selecting the point closer to the channel centre when the contour does not have a unique value at a given $x$. Using this method, they disregarded the discontinuous cores in their analyses whenever the boundary needed to be a single-valued function. Nonetheless, they repeated their analysis for the outer-enveloped core boundaries as well as including the discontinuous core instances and showed that the results are not significantly altered except for the mean core thickness, which increased by $13 \%$ using the outer-enveloped method. Here, the same inner-enveloped core boundary technique is utilized for conditional averaging and determining the spatial characteristics of the cores. Therefore, discontinuous cores are excluded from these analyses. Figure 11 shows an example of the inner-enveloped core boundary together with the parameters needed to analyse the spatial characteristics of the core, e.g. the location of the core's lower and upper boundaries ( $y_{l}$ and $y_{u}$, respectively) and centre $\left(y_{c}=\left(y_{u}+y_{l}\right) / 2\right)$, as well as the core thickness $\left(t_{c}=y_{u}-y_{l}\right)$.

\subsection{Statistical dependence of the core on inlet turbulence}

The first parameter of the flow to be analysed here is 'intermittency' $(\gamma)$, which is the time ratio that various parts of the flow spend outside of the core (Kwon et al. 2014). Figure 12(a) illustrates these profiles, indicating that the added turbulence increases the intermittency in the central regions of the channel, markedly at the centreline. Although the centreline intermittency for case $\mathrm{C}(13.5 \%)$ is approximately twice that of the REF case $(7.2 \%$, compared with $\sim 7 \%$ reported by Kwon et al. 2014), the differences between the centreline intermittency values of cases A $(12.3 \%), \mathrm{B}(12.8 \%)$ and $\mathrm{C}$ are small. The instances where the core is not present in the centre of the channel are limited to the presence of the discontinuous cores or when the core is moved above or under the centreline. To assess the latter, the location of the core boundary is inspected. Figure 12(b) illustrates p.d.f.s of the core's lower boundary $\left(y_{l}\right)$ for different test cases. This figure indicates that the probability of events where the channel core is entirely dragged above the channel centreline $\left(y_{l}>0\right)$ is equally low for the different test cases. Due to the symmetry of the channel, the same result is observed for the cases where the core upper boundary moves below the centreline (not shown here). Thus, the increased intermittency value 


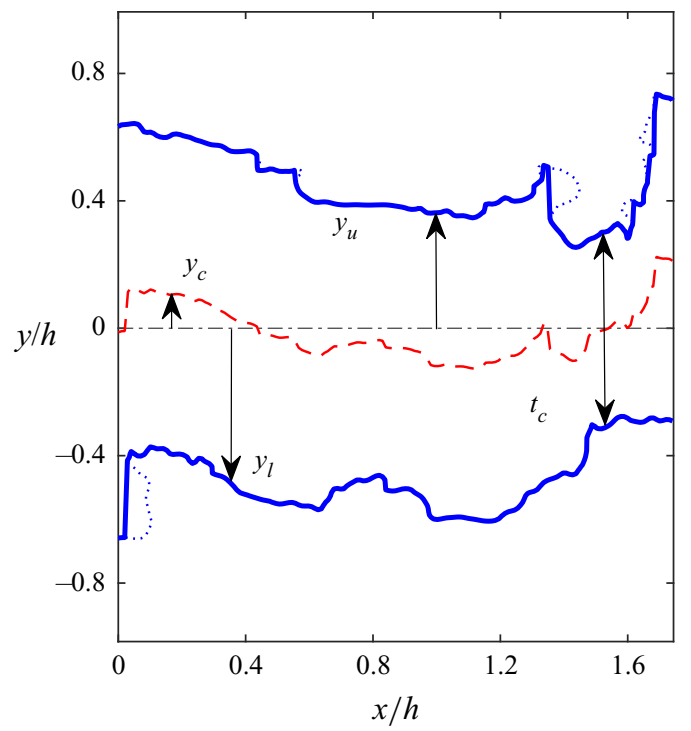

Figure 11. Enveloped (solid blue line) and un-enveloped (blue dotted line) core boundaries of the velocity field in figure 6(a). Core location and thickness parameters are depicted. The red dashed line indicates the core centre, i.e. midpoint between the upper and lower boundary. The grey dash-dot line shows the channel centreline.
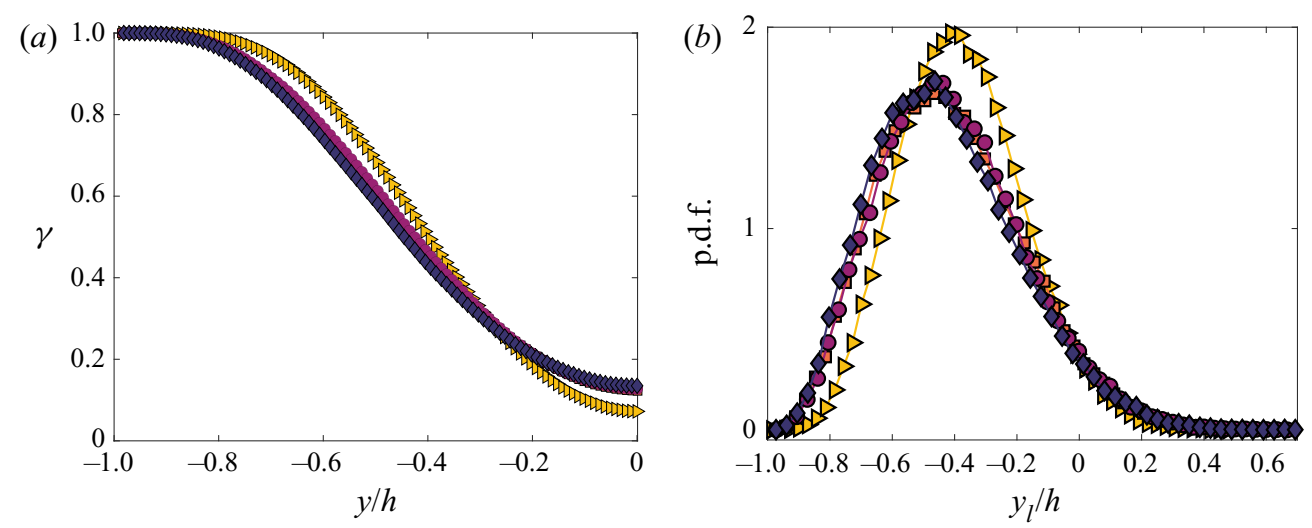

Figure 12. (a) Intermittency $(\gamma)$ profiles, and $(b)$ p.d.f. of the core's lower boundary location for cases REF

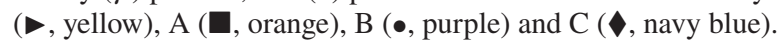

at the channel centreline, induced by the increased turbulence intensities, can be related to the more frequent presence of the discontinuous cores. This is in agreement with the information provided in table 2 .

Conversely, as is shown in figure $12(a)$, the increased turbulence intensities decrease the intermittency of the flow in the inner regions $(-0.8<y / h<-0.4)$ of the channel. At first, this may seem to contradict the previous observation regarding the increased number of discontinuous cores. To clarify, the presence of the core in the inner regions can be examined for the different cases using figure $12(b)$ and table 4 . This reveals that the added turbulence increases the probability of the core presence at the inner regions of the channel; hence, it tends to decrease the intermittency of the flow in these regions. 


\begin{tabular}{|c|c|c|c|c|c|c|c|c|}
\hline \multirow[b]{2}{*}{ Case } & \multicolumn{2}{|c|}{$y_{l} / h$} & \multicolumn{2}{|c|}{$y_{c} / h$} & \multicolumn{2}{|c|}{$t_{c} / h$} & \multicolumn{2}{|c|}{ Core mode } \\
\hline & $\mu$ & $\sigma$ & $\mu$ & $\sigma$ & $\mu$ & $\sigma$ & Symmetric $(\%)$ & Anti-symmetric (\%) \\
\hline REF & -0.378 & 0.198 & 0.004 & 0.151 & 0.761 & 0.256 & 40.0 & 60.0 \\
\hline A & -0.411 & 0.233 & 0.005 & 0.174 & 0.832 & 0.309 & 44.8 & 55.2 \\
\hline B & -0410 & 0.232 & 0.004 & 0.172 & 0.827 & 0.310 & 46.0 & 54.0 \\
\hline $\mathrm{C}$ & -0.428 & 0.234 & 0.002 & 0.170 & 0.860 & 0.317 & 47.5 & 52.5 \\
\hline
\end{tabular}

Table 4. The mean $(\mu)$ and standard deviation $(\sigma)$ of the core location parameters normalized by the channel half-height $(h)$, together with the fraction of the different core modes, i.e. symmetric and anti-symmetric, in the PIV snapshots.
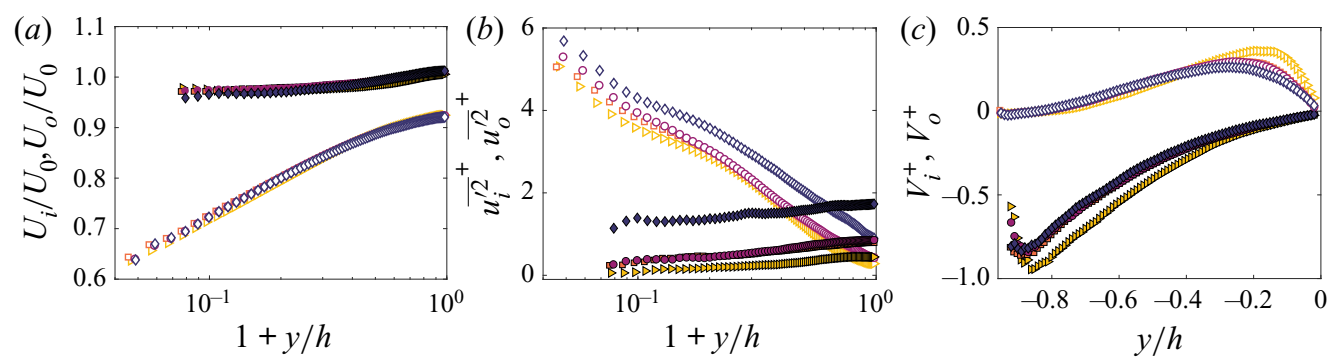

Figure 13. Weighted average profiles by $\gamma$, i.e. inside the core (symbols with black border), and by $(1-\gamma)$, i.e. outside of the core (open symbols) of $(a)$ mean streamwise velocity, $(b)$ streamwise turbulent fluctuations

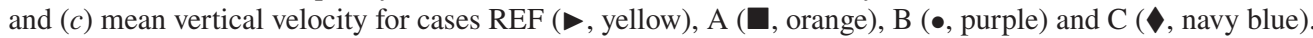

Eventually, the outcome of the two effects of the added turbulence, i.e. more frequent interruption of the core and increasing the core presence at the inner regions, which affect the intermittency in opposite ways, is a decrease in the intermittency value of the flow in the inner regions of the channel. It is noteworthy that the discrepancies between the profiles diminish in the near-wall region $(y / h<-0.8)$, where the core is seldom present, and the presence of the wall dominates the flow. Kwon et al. (2014) reported the same results for the near-wall region, where the intermittency was almost unity for all of their cases. Moreover, at the channel centreline, the same value as our REF case $(\approx 7 \%)$ was reported for the intermittency. On the other hand, they stated that increasing $R e_{\tau}$ decreases the intermittency value at any distance from the wall; however, the difference between their profiles was more distinguished in the inner regions. In other words, their intermittency profiles were essentially collapsed at the channel centreline (see Kwon et al. (2014), figure 6), indicating that the increased $R e_{\tau}$ has no major effects on the presence of the discontinuous cores or the spatial distribution of the core lower boundary at the channel centreline.

The behaviour of the flow in different wall-normal positions when it is situated inside or outside of the core is investigated next, employing a weighted averaging scheme. This scheme uses the intermittency value as the weighting parameter to zonally average the variables inside and outside the core (see Kwon et al. (2014) for details). The weighted average profiles are shown in figure 13 .

Figure 13(a) depicts the weighted average streamwise velocity profiles inside $\left(U_{i}\right)$ and outside $\left(U_{o}\right)$ the core normalized by the channel centreline mean velocity. As shown, the averaged flow velocity monotonically scales with the centreline mean velocity inside the core at every wall-normal position for all cases. This happens for the active cases 


\section{Effect of inlet turbulence on a channel's quiescent core}

as well, demonstrating that the mean velocity of the flow inside the core is always the same, irrespective of the presence of LMCs and HMCs. This will be further elaborated on in the next section. Figure 13(a) also suggests that the added turbulence does not change the zonally averaged streamwise velocity profile outside the core. The distinct behaviour of the zonally averaged velocity profile inside and outside the core, as well as their invariance, is reminiscent of the two-state model introduced by Krug, Philip \& Marusic (2017) suggesting that the discrepancies observed in the mean velocity profiles (figure $3 a$ ) are essentially due to the difference in fluctuations of the core boundary inside the channel.

The inner-normalized streamwise turbulent fluctuations are zonally averaged inside

$\left({\overline{u_{i}^{\prime 2}}}^{+}\right)$and outside $\left({\overline{u_{o}^{\prime}}}^{+}\right)$the core using the same method, which highlights the deviations of the streamwise velocity from the weighted average velocity. Figure 13(b) shows the resulting profiles for different test cases. This figure, in contrast to figure 13(a), identifies an apparent difference between the interior profiles of different cases, as well as the exterior ones. This indicates that increasing the turbulence level intensifies the streamwise fluctuations in the core, which is directly related to the presence of the LMC and HMC. This will be further detailed in the next section. The average turbulence level inside the core is relatively insensitive to the wall-normal position, at least when compared with the turbulence outside of the core; the latter follows a profile not so dissimilar to the mean variance profile of figure $3(b)$. Weighted average wall-normal velocity profiles are illustrated in figure $13(c)$. The eye is immediately drawn to the peaks and troughs. The vertical velocity inside the core is negative in the bottom half of the channel, suggesting that the flow inside the core tends to move towards the wall. On the other hand, the flow outside the core is associated with a positive wall-normal velocity, which indicates that the flow tends to move towards the core when it is situated outside of it. A close look at the peaks and troughs of the curves suggests that the increased turbulence level appears to dampen this relationship, diminishing bulk vertical transport processes.

Figure 14 shows the distribution of the centre location and thickness of the core while table 4 lists the mean $(\mu)$ and standard deviation $(\sigma)$ parameters. As shown in figure 14(a), the location of the core centre has a normal distribution, which is not changed considerably under the influence of the added turbulence. The mean value is almost zero for all cases (table 4), indicating it is positioned on the channel centreline. This was expected due to the symmetry of the channel. Moreover, figure 14(a) points to the fact that even though the added turbulence pushes the lower boundary of the core towards the wall more frequently (figure 12b), it does not affect the distribution of the centre of the core, thus requiring the core thickness to increase. This claim is confirmed by both figure 14(b) and table 4, which show an increasing trend in the mean thickness of the core with increasing turbulence intensity. Kwon et al. (2014) had similarly observed an increase in the mean core thickness with increasing $\operatorname{Re}_{\tau}$.

Kwon et al. (2014) analysed the correlations between the fluctuations of the core's lower and upper boundary locations $\left(S_{y}=\left(y_{l}-\overline{y_{l}}\right)\left(y_{u}-\overline{y_{u}}\right)\right)$ to assess the symmetry of the cores with respect to the channel centreline. Averaging the correlation parameter $\left(S_{y}\right)$ for each velocity field, they stated that in the majority of the velocity fields (approximately $70 \%$ of their PIV frames), the core had an anti-symmetric mode $\left(\overline{S_{y}}>0\right)$ insensitive to $R e_{\tau}$. This was in agreement with the observations of Teitel \& Antonia (1990) and Jiménez et al. (2010) regarding the anti-symmetry of the large-scale structures in a turbulent channel flow. In order to explore the influence of the inlet turbulence intensity on the symmetry of the core, the same correlation parameter $\left(S_{y}\right)$ is calculated and averaged for the velocity fields of different test cases. Table 4 provides the results and shows a 

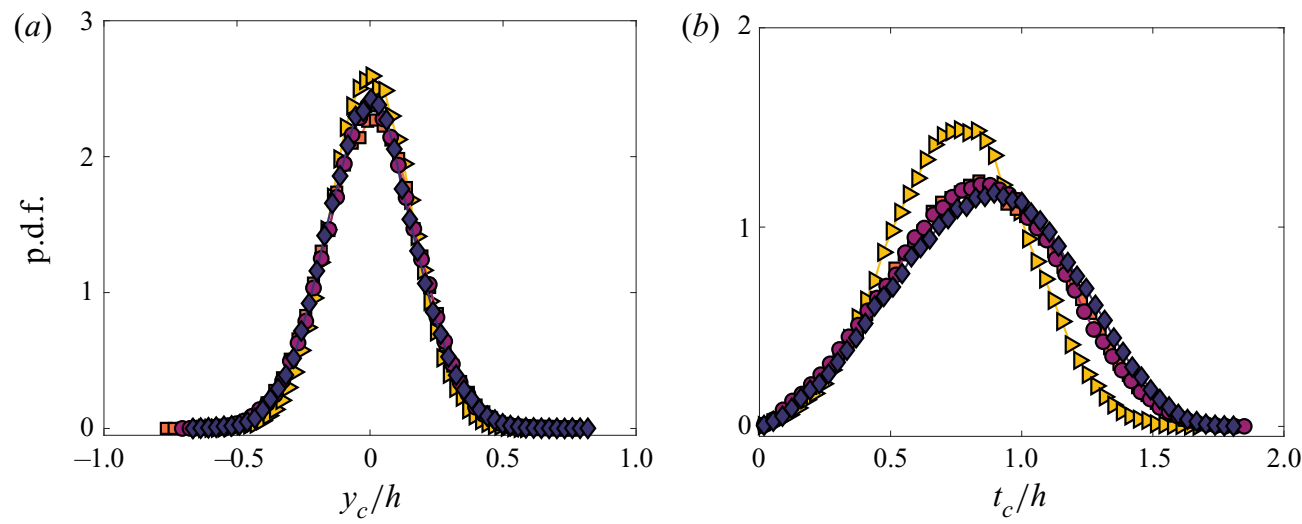

Figure 14. p.d.f. of $(a)$ the core's centre location and $(b)$ core thickness for cases REF ( $\bullet$, yellow), A ( orange), B $(\bullet$, purple) and C ( $\downarrow$, navy blue $)$.

trend towards more symmetric cores with increasing the centreline turbulence intensity. One possible explanation is that the added turbulence tends to homogenize the flow, which in turn increases the symmetry of the instantaneous core structure with respect to the channel centreline. In fact, increased turbulence intensity is known to homogenize flows by increasing turbulent transport, e.g. reducing the vortex shedding effects behind an axisymmetric disk (Rind \& Castro 2012) and enhancing wake recovery behind a wall-mounted cube (Hearst, Gomit \& Ganapathisubramani 2016).

The flow characteristics in the vicinity of the core boundary are also of great interest. Conditional averaging across the core boundary is employed here, where the origin of the coordinate system is translated to the core's lower boundary. Moving along the lower core boundary, the variables are averaged towards the core centre and the lower wall, i.e. $\left(y-y_{l}\right)>0$, and $\left(y-y_{l}\right)<0$, respectively. Conditionally averaged profiles are presented in figure 15 , where the angle brackets, \langle\rangle , denote conditional averaging along the core. Figure 15(a) shows conditionally averaged streamwise velocity profiles. Due to a difference in the core boundary thresholds, the streamwise velocities are offset by the local threshold value of the core boundary and then conditionally averaged, i.e. $\left\langle u-u_{t h}\right\rangle$. This makes the velocity profiles comparable in a similar frame of reference. All profiles experience a sharp jump in the streamwise velocity across the core boundary. As mentioned before, this is typical of a UMZ and shows a unified streamwise velocity value inside the core, which is separated from that outside of it by a thin shear layer (cf. Kwon et al. 2014; Yang et al. 2016). The four curves show the same behaviour inside and outside the core suggesting that the shape of the average velocity profile is robust to the changes in turbulence level. However, the fitted lines to the curves in the vicinity of the core boundary demonstrate that the maximum slope slightly increases with increasing centreline turbulence intensity. Furthermore, the velocity jump across the core boundary also increases with an increase in centreline turbulence intensity. Kwon et al. (2014) found that the jump in velocity across the core boundary $\left(0.06 U_{0}\right)$ is invariant with $U_{\tau}$ for canonical turbulent channel flow. The velocity jump is quantified here with linear fits to the profiles inside and outside the core region. The resulting velocity jumps were measured as $D\left[U_{0}\right] \approx 0.064,0.071,0.075$, and 0.077 , for cases REF, A, B and C, respectively, which shows an increase with increasing centreline turbulence intensity. This notion is similar to the previous observation of Hearst et al. (2021) that the velocity jump across the upper-most UMZ edge of a ZPG-TBL increases with increasing FST intensity. 

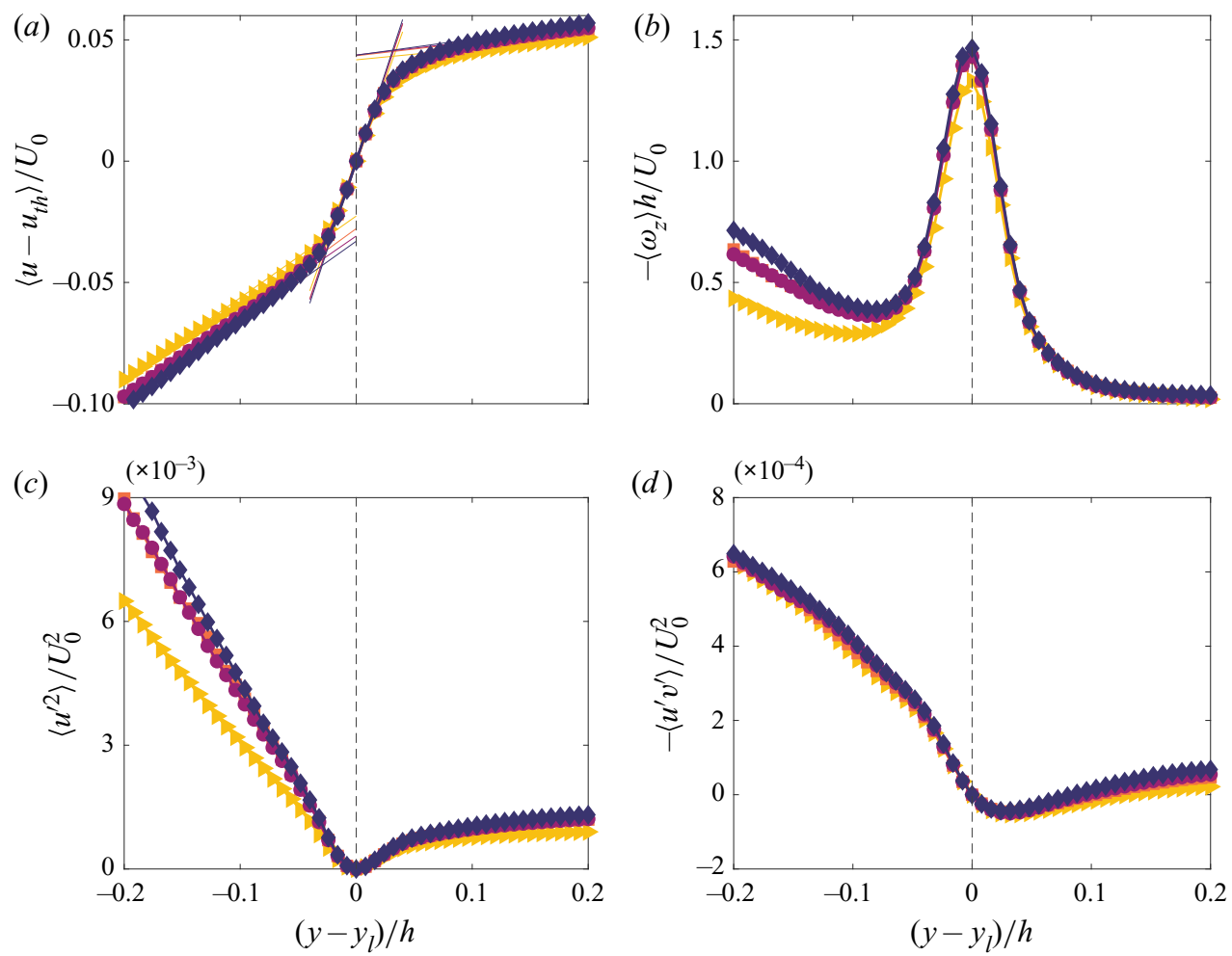

Figure 15. Profiles of (a) streamwise velocity, $(b)$ out of plane vorticity, $(c)$ streamwise turbulent fluctuations and $(d)$ Reynolds shear stress, conditionally averaged across the core boundary for cases REF ( $>$, yellow), A

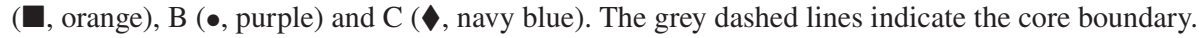

Figure $15(b)$ presents the conditionally averaged vorticity profiles, where increased values of the clockwise ('prograde') vorticity on the bottom wall are observed outside of the core for increased turbulence intensities. This effect holds all the way to the core boundary, where the vorticity profile exhibits a peak whose value slightly increases with the inlet turbulence intensity. The latter is in line with the increased jump in velocity caused by an increased turbulence level. On the other hand, the vorticity profiles collapse reasonably well inside the core region. As expected for the QC, the vorticity value is negligibly small inside the core away from the boundary.

Figure 15(c) shows the streamwise fluctuations conditionally averaged across the core boundary. Discrepancies exist between the profiles outside of the core, while an almost equally low turbulence level is observed for different cases inside the core. The latter may seem contradictory to what was observed in figure 13(b); however, one should pay attention to the different averaging schemes. The weighted average method evaluates the streamwise velocity fluctuations in different locations within the channel relative to the zonally averaged velocity. In contrast, the conditional-averaging scheme assesses the fluctuations of the streamwise velocity offsetting the velocities by the local boundary threshold value. Further details are presented in the next section, where different types of cores are compared with one another. Finally, figure $15(d)$ shows the conditionally averaged Reynolds shear stress profiles, indicating similar levels of turbulent shear stress for all the cases irrespective of turbulence intensity. This was expected given that the Reynolds shear stress profiles in figure $3(b)$ were not affected by the inlet turbulence. 

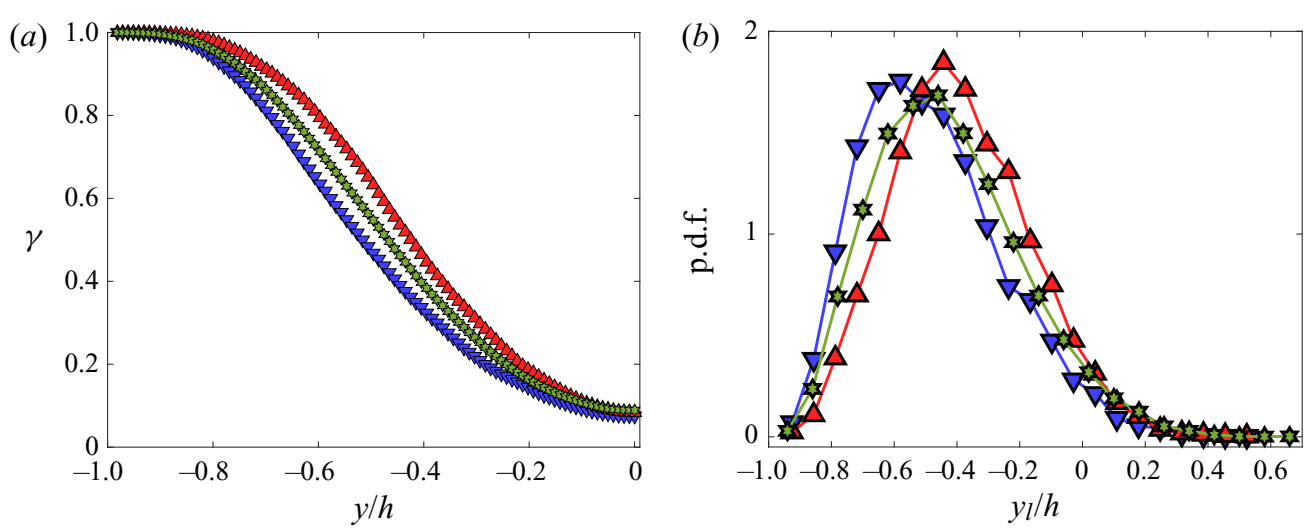

Figure 16. (a) Intermittency $(\gamma)$ profiles, and $(b)$ p.d.f. of the core's lower boundary location for LMCs $(\boldsymbol{\nabla}$, blue), OCs ( $\star$, green) and HMCs $(\boldsymbol{\Lambda}$, red $)$ of case C.

\subsection{Statistical comparison of the different core states}

As was shown in $\S 4.2$, increasing the turbulence level inside the channel by increasing the inlet turbulence yields the formation of the new core states. The core states differ from one another in the boundary threshold value, which also implies different levels of momentum for each core. The cores were labelled LMC, OC and HMC corresponding to their momentum. Here, the characteristics of the new core states are explored in order to determine how they contribute to the total picture presented above. Case $\mathrm{C}$ is used as the example for this section as it has the highest number of cores in each state. The discontinuous cores are disregarded throughout this section.

Figure 16(a) shows the intermittency profiles for different core states. Due to the exclusion of the discontinuous cores, all the profiles roughly match at the channel centreline. Moving from the centreline towards the wall, the profile of the OCs is flanked by that of the LMCs and HMCs $(-0.8<y<-0.3)$, where the flow spends the most time inside the core when they are LMCs, and the least when they are HMCs. Figure 16(b) shows the p.d.f.s of the core lower boundary location. An increase in the momentum level shifts the p.d.f. to the right, explaining the trends observed in figure 16(a). The cores are barely present in the near-wall region $(y / h<-0.9)$ regardless of the core state.

The same weighted average scheme as above is employed here to explore the behaviour of the flow inside and outside of the cores. Figure 17(a) shows the weighted average streamwise velocity profiles for different core states. As illustrated, different levels of velocity are present inside and outside the cores. When the flow is located inside the 'ordinary' core, its average velocity scales with the centreline mean velocity. Alternatively, the characteristic velocity is $0.9 U_{0}$ and $1.1 U_{0}$ for the LMCs and HMCs, respectively. The same trend is also observed for the zonally averaged velocity profiles out of the cores. In contrast to figure $13(a)$ where the zonally averaged profiles collapsed for different test cases, figure 17(a) emphasizes the different momentum levels of each core state. Nevertheless, in the cumulative averaging, the LMCs and HMCs roughly cancel each other out.

Figure $17(b)$ depicts the zonally averaged streamwise fluctuation profiles inside and outside the core for different core states. Whereas figure 17(a) showed different average velocity levels for the flow inside the core, figure 17(b) indicates that the streamwise fluctuations of the flow when it is situated inside the core are uniformly low regardless of the wall-normal position as well as the core state. This may seem contradictory to the 

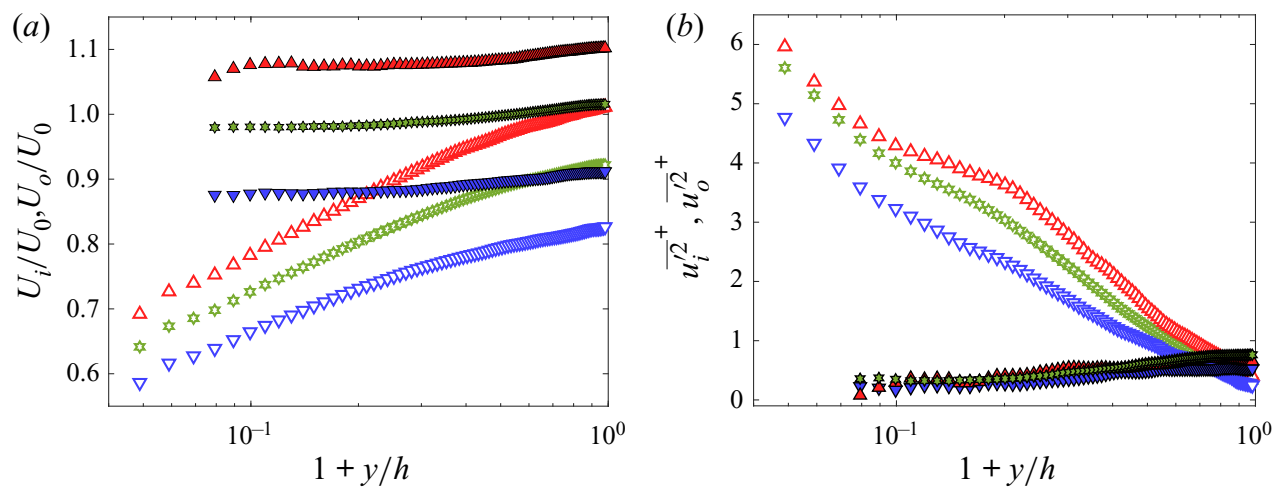

Figure 17. Weighted average profiles by $\gamma$, i.e. inside the core (symbols with black border), and by $(1-\gamma)$, i.e. out of the core (open symbols) of $(a)$ mean streamwise velocity, and $(b)$ streamwise turbulent fluctuations for LMCs ( $\mathbf{\nabla}$, blue), OCs ( $\star$, green) and HMCs $(\boldsymbol{\Lambda}$, red) of case C.
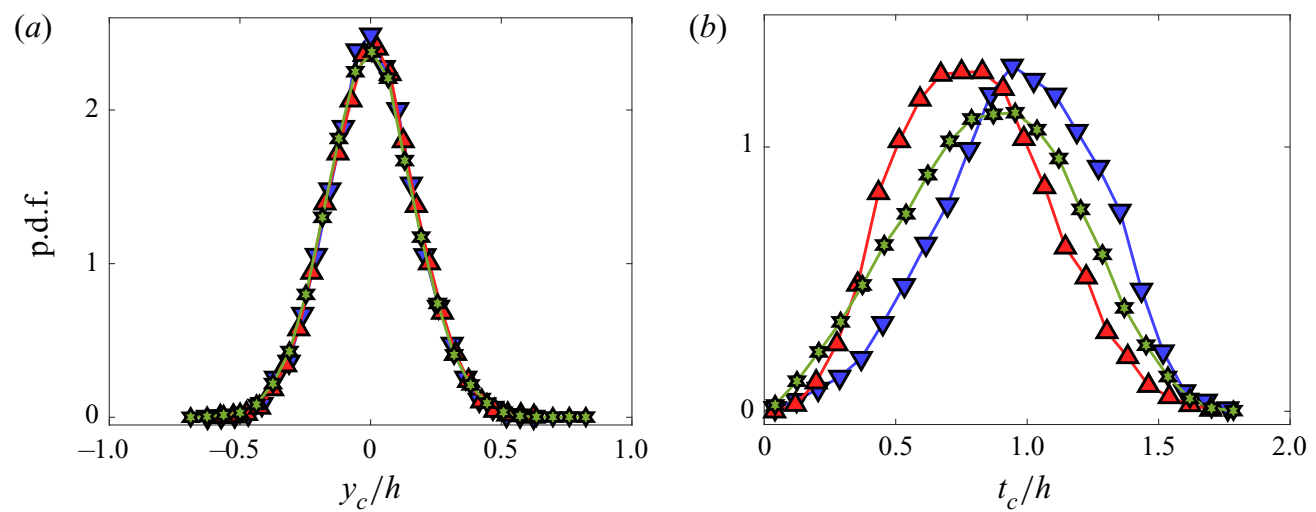

Figure 18. p.d.f. of $(a)$ the core's centre location and $(b)$ core thickness for LMCs ( $\mathbf{\nabla}$, blue), OCs $(\star$, green) and HMCs $(\boldsymbol{\Lambda}$, red) of case C.

relatively high level of fluctuations in case $\mathrm{C}$ observed in figure 13(b). However, here the deviations of the streamwise velocity from the zonally averaged velocity are calculated for each core separately. In other words, LMCs and HMCs are responsible for the increased fluctuation level of case $\mathrm{C}$ observed in figure 13(b) since they have a distinctively different velocity level than the weighted average velocity.

Figure 18 illustrates the distribution of the centre location and thickness of the cores. The mean and standard deviation values are presented in table 5. Figure 18(a) illustrates a normal distribution for the core's centre location around the channel centreline for all three cores. This is a result of the symmetry of the channel. Figure $18(b)$ and table 5 complement the trend observed in figure $16(b)$ and show an increase in the thickness of the cores with a decrease in their momentum level. Thus, LMCs are thicker than the OCs and reside closer to the walls, whereas the HMCs are thinner than the OCs and usually situated in the central regions of the channel. The information provided in table 5 does not show a clear trend for the core symmetry with their momentum level.

Conditionally averaged profiles across the core boundary are illustrated in figure 19 for the different core states. Figure 19(a) shows conditionally averaged velocity profiles, where the profiles are approximately collapsed both in and out of the core region. 


\begin{tabular}{|c|c|c|c|c|c|c|c|c|}
\hline \multirow[t]{2}{*}{ Core State } & \multicolumn{2}{|c|}{$y_{l} / h$} & \multicolumn{2}{|c|}{$y_{c} / h$} & \multicolumn{2}{|c|}{$t_{c} / h$} & \multicolumn{2}{|c|}{ Core mode } \\
\hline & $\mu$ & $\sigma$ & $\mu$ & $\sigma$ & $\mu$ & $\sigma$ & Symmetric (\%) & Anti-symmetric (\%) \\
\hline LMC & -0.479 & 0.226 & 0.005 & 0.165 & 0.967 & 0.298 & 44.5 & 55.5 \\
\hline OC & -0.427 & 0.236 & 0.001 & 0.171 & 0.855 & 0.321 & 48.2 & 51.8 \\
\hline HMC & -0.389 & 0.219 & 0.008 & 0.166 & 0.794 & 0.281 & 40.6 & 59.4 \\
\hline
\end{tabular}

Table 5. The mean $(\mu)$ and standard deviation $(\sigma)$ of the core location parameters normalized by the channel half-height $(h)$, together with the fraction of the different core modes, i.e. symmetric and anti-symmetric, in the PIV snapshots.
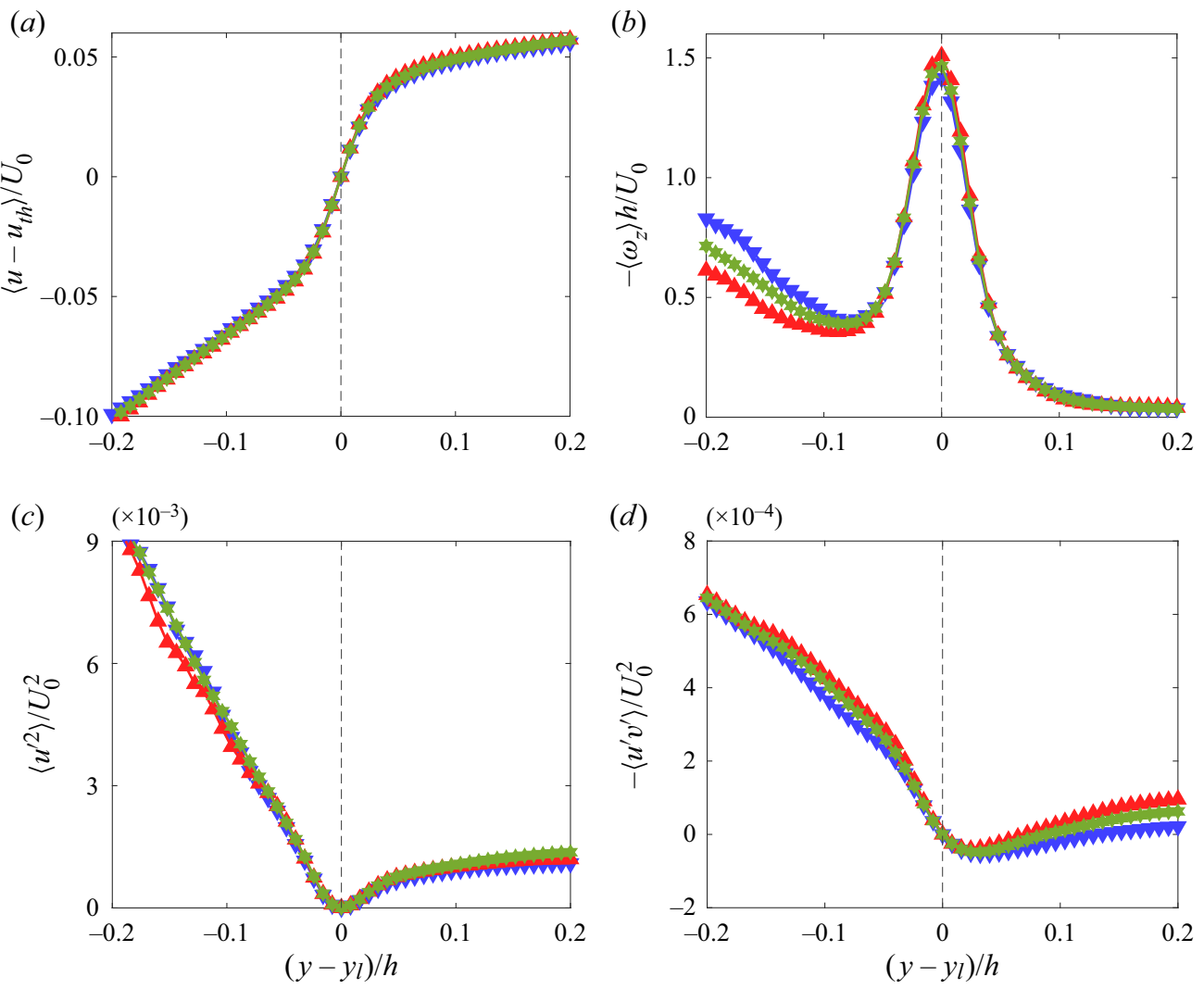

Figure 19. Profiles of $(a)$ streamwise velocity, $(b)$ out of plane vorticity, (c) streamwise turbulent fluctuations and $(d)$ Reynolds shear stress, conditionally averaged across the core boundary for LMCs ( $\mathbf{\nabla}$, blue), OCs ( $\star$, green) and HMCs ( $\boldsymbol{\Lambda}$, red) of case C. The grey dashed line indicates the core boundary.

This occurs because the profiles are offset by the local boundary threshold velocity $\left(u_{t h}\right)$. The same sharp jump in the streamwise velocity at the core boundary, as shown in figure $15(a)$, is visible for all three types of cores. This figure confirms that all the core states are indeed uniform momentum zones which differ in the core boundary threshold value and hence the characteristic velocity of the zone. Figure 19(b) depicts the conditionally averaged vorticity across the core boundary for different core states. As in figure $15(b)$, a peak is present at the core boundary location. Approaching the core's 


\section{Effect of inlet turbulence on a channel's quiescent core}

central regions, the profiles collapse. Once more, a discrepancy is exhibited outside the core region. The higher vorticity for the LMCs was because the area under the interface was closer to the wall where there is high vorticity. Figures $19(c)$ and $19(d)$ show Reynolds streamwise normal and shear stress conditionally averaged across the core boundary, respectively. The turbulence level inside the core region is uniformly low, which is typical of UMZs. This might seem to contrast with the trends observed in figure 13(b). However, one should note that if the reference frame is fixed inside the channel, the passage of different cores with different velocity levels implies a more turbulent state compared with the REF case where all cores have the same characteristic velocity. Now, suppose the streamwise fluctuations are assessed with respect to the local streamwise velocity of the core, i.e. the reference frame moves with the core boundary, and the velocities are offset by the local boundary threshold value. In such a case, the area inside the core will look quiescent regardless of the core state, i.e. LMC, OC or HMC. This corroborates the fact that each core is a localized UMZ. Although the identification of the new core states was tentative at first, the aforementioned observations affirm the presence of the new core states for increased turbulence intensities and indicate the reliability of the core identification procedure.

\section{Conclusions}

PIV measurements were carried out to investigate the effects of the inlet turbulence on the core of a turbulent channel flow as well as the turbulence statistics. Various inlet turbulence levels were produced utilizing an active grid located at the inlet of an air channel. Matching the centreline mean velocity $\left(U_{0} \approx 10 \mathrm{~m} \mathrm{~s}^{-1}, R e_{H} \approx 33200\right)$ for different test cases resulted in no apparent correlation between $U_{\tau}$ and inlet turbulence levels studied here; in contrast to previous studies on the effects of FST on a ZPG-TBL (Blair 1983a; Hancock \& Bradshaw 1983; Sharp et al. 2009; Esteban et al. 2017; Jooss et al. 2021). Consequently, $U_{\tau}$ was approximately matched for different cases $\left(U_{\tau} \approx 0.47 \mathrm{~m} \mathrm{~s}^{-1}, R e_{\tau} \approx 770\right)$. It was shown that the log layer in the mean velocity profile of a turbulent channel flow is not considerably affected by varying the centreline turbulence intensity, whereas the wake region was suppressed for increased turbulence levels. Moreover, significant increase in the streamwise fluctuations was observed in the outer regions with increasing turbulence intensity. These observations are in agreement with previous observations for a ZPG-TBL subjected to FST (Blair 1983b; Hancock \& Bradshaw 1983; Thole \& Bogard 1996; Sharp et al. 2009; Dogan et al. 2016, 2019; Jooss et al. 2021).

The central UMZ of the channel flow was identified utilizing a modification to the techniques used earlier by Kwon et al. (2014), Yang et al. (2016) and Jie et al. (2019, 2021). In the present procedure, p.d.f.s of the highest and the second highest modal velocity were plotted separately, resulting in a more evident boundary threshold value. The number of discontinuous cores was found to scale with turbulence intensity. Two new threshold values of the core boundary were also found for each of the elevated turbulent cases and were employed together with the canonical boundary threshold value to correctly demarcate the core region of the channel flow. Consequently, in addition to the conventional continuous core of the channel (OC), two new core states were identified, referred to as LMC and $\mathrm{HMC}$, whose occurrence was found to scale with the centreline turbulence intensity. Zonal averaging revealed an increasing trend in the variance of the streamwise velocity for the flow situated inside the core region with increased inlet turbulence intensities. However, conditional averaging across the core boundary indicated matched levels of local turbulent fluctuations inside the cores of the different test cases. Furthermore, analysing the spatial characteristics of the cores revealed that the inlet turbulence increases the thickness of 


\begin{tabular}{lcccc} 
Case & \multicolumn{4}{c}{ No. of cores (\%) } \\
\cline { 2 - 5 } & low & ordinary & high & Discontinuous \\
REF & 0 & 96.4 & 0 & 3.6 \\
A & 1.7 & 88.4 & 2.1 & 7.8 \\
B & 2.2 & 83.7 & 2.6 & 8.5 \\
C & 10.0 & 68.6 & 10.7 & 10.7
\end{tabular}

Table 6. Presence of the different core types in the velocity fields of each test case at $X / H=100$ as a percentage of the total fields (2000).

the core and moves the core boundary towards the wall. The latter might be considered similar to the effect of FST on a ZPG-TBL in moving the upper boundary of the topmost UMZ closer to the wall (Hearst et al. 2021). Moreover, it is found that the increased inlet turbulence levels lead to an increase in the symmetric core events, which may be related to the tendency of the added turbulence to homogenize the flow.

Comparing the characteristics of the new core states (LMC and HMC) with those of the OC indicated significant differences in the spatial characteristics, where the LMCs were generally thicker than the OCs and their boundary was situated closer to the wall, whereas the boundary of the HMCs commonly reside closer to the channel centreline than that of the OCs; thus they are generally thinner than the OCs. Nonetheless, the mean turbulence statistics were identical inside the core region, e.g. uniform velocity levels and low turbulence intensities, underlining the analogous essence of the cores, i.e. the cores are UMZs.

To conclude, the results of this study show that inlet turbulence has a significant impact on the core of the turbulent channel flow. It influences the size of the core and the frequency it appears in various states with different momentum levels. The permanence of the core, although it takes on different forms, suggests that it remains a driving force in the dynamics of fluid motion in internal flows, even when the inlet is far from the idealized canonical case.

Acknowledgements. The authors would like to thank Mr L. Li who aided with the assembly of the air channel.

Funding. This project and all authors were funded by the Research Council of Norway (RCN) project no. 280578 (DiHI-Tech). M.A. and R.J.H. were also funded by RCN project no. 288056 (WallMix).

Declaration of interests. The authors report no conflict of interest.

Author ORCIDs.

(1) Masoud Asadi https://orcid.org/0000-0003-2336-1901;

D. Jason Hearst https://orcid.org/0000-0003-2002-8644.

\section{Appendix A. Core status at $X / H=100$}

Table 6 lists the number of different core types identified for different test cases using the auxiliary PIV results performed $100 H$ downstream of the inlet. Comparing these results with those of table 2 indicates a general agreement in trends, while the discrepancies are generally less than $1 \%$ and deemed to be acceptable considering the lower number of PIV frames at $X / H=100$. In addition, performing the same statistical analysis on the auxiliary data (not shown here) confirms the current observations. 


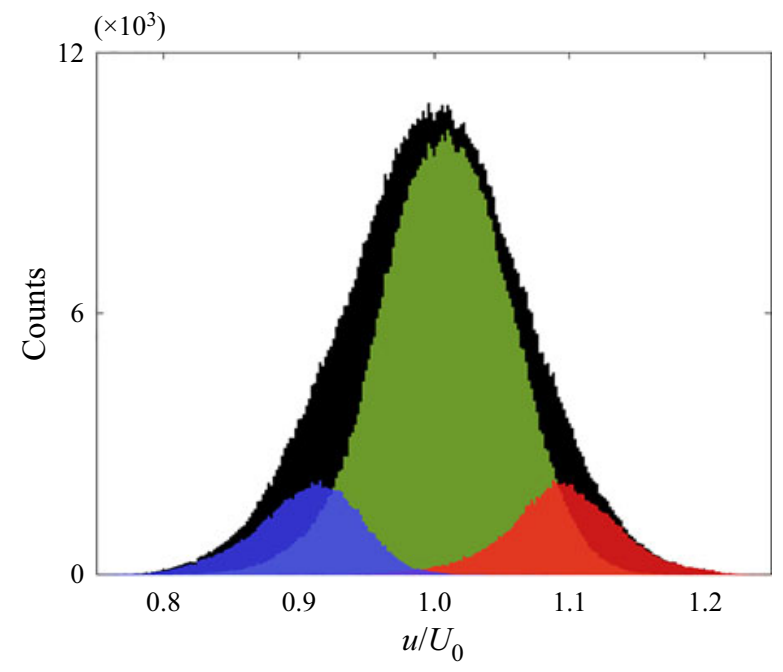

Figure 20. Histograms of the instantaneous streamwise centreline velocity of case $\mathrm{C}$ for all velocity fields (black), and the velocity fields with an OC (green), LMC (blue) or HMC (red).

\section{Appendix B. Sensitivity analysis for the HMC identification process}

As stated in $\$ 4.2$, the velocity fields containing an HMC are not easily identifiable, unlike the LMC fields. In the first step of the employed method, a presumed threshold value higher than the threshold of the OCs is utilized to find the velocity fields with continuous core boundaries as the possible candidates for the HMC instances. Although it may seem arbitrary, the threshold value was chosen such that the number of detected fields for the most turbulent case (C) was approximately the same as their LMC counterparts. Furthermore, no HMC instances were identified for the REF case using this threshold value, which was expected since REF does not include any LMC instances either. Figure 20 shows histograms of the instantaneous streamwise velocity at the channel centreline for all fields of case $\mathrm{C}$, as well as the velocity fields possessing an OC, LMC, or HMC. As expected, the instantaneous velocity at the channel centreline has a normal distribution around its mean value. Moreover, the histograms of the LMC and HMC fields are situated symmetrically at the tails of the cumulative histogram, representing the lowand high-momentum levels of these cores, respectively. Therefore, figure 20 confirms that the identified HMC fields are counterparts for the LMCs. These two counterparts are symmetrically distributed around the OCs. It should be noted that the peak in the histogram of OC has a small offset from the global peak $\left(U_{0}\right)$ due to the exclusion of the fields with a discontinuous core.

Varying the above-mentioned presumed threshold value changes the number of identified fields. Different presumed threshold values are tested for case $\mathrm{C}$ to investigate the sensitivity of the HMC identification process and the results. Table 7 lists the tested values and the number of the identified fields, as well as the resulting threshold value. Increasing the presumed threshold value decreases the number of the identified fields as this chooses the fields with more substantial fluctuations of the streamwise velocity; on the other hand, the opposite is true for decreased values of the presumed threshold. Consequently, the resulting threshold value is slightly higher for the increased presumed threshold value, whereas it is found to be slightly lower for decreased presumed threshold values. It should be noted that further decreasing the presumed threshold value significantly biases the 
Presumed threshold value No. of identified fields (\%) Identified threshold value

$\begin{array}{lrr}1.035 U_{0} & 25.0 & 1.01 U_{0} \\ 1.065 U_{0} & 11.4 & 1.04 U_{0} \\ 1.095 U_{0} & 4.4 & 1.06 U_{0} \\ 1.125 U_{0} & 1.3 & 1.09 U_{0}\end{array}$

Table 7. Number of the identified 'HMC instances by different presumed threshold values as a percentage of the total fields (5100) for case $\mathrm{C}$, together with the threshold values identified by the p.d.f. analysis of those fields.
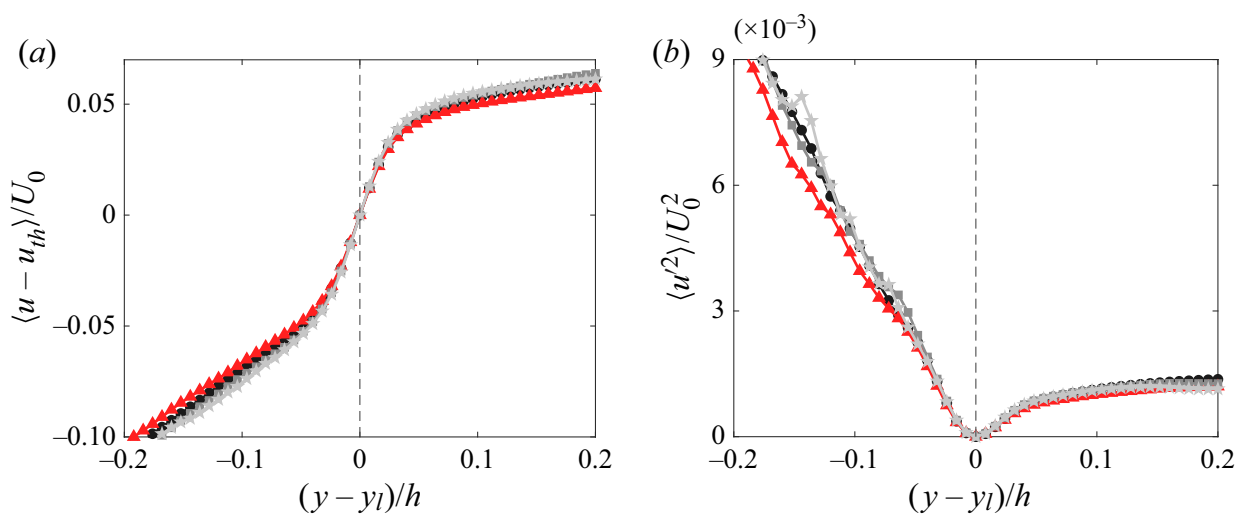

Figure 21. Profiles of streamwise $(a)$ velocity, and (b) turbulent fluctuations, conditionally averaged across the core boundary for HMC fields identified by a presumed threshold value of $1.035 U_{0}(\bullet$, black $), 1.065 U_{0}(\boldsymbol{\Delta}$, red), $1.095 U_{0}\left(\square\right.$, dark grey) and $1.125 U_{0}(\star$, light grey). The dashed line indicates the core boundary.

HMC identification process by choosing a substantial number of the ordinary fields, e.g. a presumed threshold value of $1.005 U_{0}$ picks approximately $27 \%$ of the PIV frames of the REF case as HMC instances, while this number is approximately $1 \%$ for the lowest threshold value listed in table 7 and $0 \%$ for the threshold value used in the analysis $\left(1.065 U_{0}\right)$. On the other hand, further increase of this value causes no field to be identified as a HMC instance.

Figure 21 shows the conditionally averaged streamwise velocity and turbulent fluctuations for the identified fields with the new threshold value. A presumed threshold value of $1.065 U_{0}$ yields the most uniform average velocity and the least turbulent fluctuations inside the core region; however, the other values of the presumed threshold do not affect the results significantly. Hence, the trends observed in this study for HMCs are robust to the presumed threshold value used to identify these fields and even if other thresholds had been employed, the general trends would not change.

\section{REFERENCES}

Adrian, R.J., Meinhart, C.D. \& Tomkins, C.D. 2000 Vortex organization in the outer region of the turbulent boundary layer. J. Fluid Mech. 422, 1-54.

Bernardini, M., Pirozzoli, S. \& Orlandi, P. 2014 Velocity statistics in turbulent channel flow up to $R e_{\tau}=4000$. J. Fluid Mech. 742, 171-191.

BLAIR, M.F. $1983 a$ Influence of free-stream turbulence on turbulent boundary layer heat transfer and mean profile development. Part 1. Experimental data. Trans. ASME J. Heat Transfer 105 (1), 33-40. 


\section{Effect of inlet turbulence on a channel's quiescent core}

BLAIR, M.F. $1983 b$ Influence of free-stream turbulence on turbulent boundary layer heat transfer and mean profile development. Part 2. Analysis of results. Trans. ASME J. Heat Transfer 105 (1), 41-47.

CASTRO, I.P. 1984 Effects of free stream turbulence on low Reynolds number boundary layers. J. Fluids Engng 106 (3), 298-306.

Chen, X., Chung, Y.M. \& Wan, M. 2020 Uniform-momentum zones in a turbulent pipe flow. J. Fluid Mech. 884, A25.

Chen, X., Chung, Y.M. \& Wan, M. 2021 The uniform-momentum zones and internal shear layers in turbulent pipe flows at Reynolds numbers up to $R_{\tau}=1000$. Intl J. Heat Fluid Flow 90, 108817.

Comte-Bellot, G. \& CoRRsin, S. 1966 The use of a contraction to improve the isotropy of grid-generated turbulence. J. Fluid Mech. 25 (4), 657-682.

Dogan, E., Hanson, R.E. \& GANAPAThisubramani, B. 2016 Interactions of large-scale free-stream turbulence with turbulent boundary layers. J. Fluid Mech. 802, 79-107.

Dogan, E., Hearst, R.J. \& Ganapathisubramani, B. 2017 Modelling high Reynolds number wall-turbulence interactions in laboratory experiments using large-scale free-stream turbulence. Phil. Trans. R. Soc. A 375 (2089), 20160091.

Dogan, E., Hearst, R.J., Hanson, R.E. \& Ganapathisubramani, B. 2019 Spatial characteristics of a zero-pressure-gradient turbulent boundary layer in the presence of free-stream turbulence. Phys. Rev. Fluids 4 (8), 084601.

Eisma, J., Westerweel, J., Ooms, G. \& Elsinga, G.E. 2015 Interfaces and internal layers in a turbulent boundary layer. Phys. Fluids 27 (5), 055103.

Ertunç, Ö., Özyilmaz, N., Lienhart, H., Durst, F. \& Beronov, K. 2010 Homogeneity of turbulence generated by static-grid structures. J. Fluid Mech. 654, 473-500.

Esteban, L.B., Dogan, E., Rodríguez-López, E. \& Ganapathisubramani, B. 2017 Skin-friction measurements in a turbulent boundary layer under the influence of free-stream turbulence. Exp. Fluids 58 (9), 115.

FAN, D., XU, J., YAO, M.X. \& HickeY, J.-P. 2019 On the detection of internal interfacial layers in turbulent flows. J. Fluid Mech. 872, 198-217.

GRAHAM, J., et al. 2016 A web services accessible database of turbulent channel flow and its use for testing a new integral wall model for LES. J. Turbul. 17 (2), 181-215.

Gul, M., Elsinga, G.E. \& Westerweel, J. 2020 Internal shear layers and edges of uniform momentum zones in a turbulent pipe flow. J. Fluid Mech. 901, A10.

HANCOCK, P.E. \& BRADSHAW, P. 1983 The effect of free-stream turbulence on turbulent boundary layers. J. Fluids Engng 105 (3), 284-289.

HANCOCK, P.E. \& BRADSHAW, P. 1989 Turbulence structure of a boundary layer beneath a turbulent free stream. J. Fluid Mech. 205, 45-76.

Hearst, R.J., Dogan, E. \& Ganapathisubramani, B. 2018 Robust features of a turbulent boundary layer subjected to high-intensity free-stream turbulence. J. Fluid Mech. 851, 416-435.

Hearst, R.J., Gomit, G. \& Ganapathisubramani, B. 2016 Effect of turbulence on the wake of a wall-mounted cube. J. Fluid Mech. 804, 513-530.

Hearst, R.J. \& LAvoie, P. 2015 The effect of active grid initial conditions on high Reynolds number turbulence. Exp. Fluids 56 (10), 185.

Hearst, R.J., de Silva, C.M., Dogan, E. \& Ganapathisubramani, B. 2021 Uniform-momentum zones in a turbulent boundary layer subjected to freestream turbulence. J. Fluid Mech. 915, A109.

HoyAs, S. \& JiménEZ, J. 2006 Scaling of the velocity fluctuations in turbulent channels up to $R e_{\tau}=2003$. Phys. Fluids 18 (1), 011702.

IsAZA, J.C., SAlazAR, R. \& WARhAFT, Z. 2014 On grid-generated turbulence in the near- and far field regions. J. Fluid Mech. 753, 402-426.

Jie, Y., Andersson, H.I. \& ZHAO, L. 2021 Effects of the quiescent core in turbulent channel flow on transport and clustering of inertial particles. Intl J. Multiphase Flow 140, 103627.

Jie, Y., Xu, C., Dawson, J.R., Andersson, H.I. \& ZHAO, L. 2019 Influence of the quiescent core on tracer spheroidal particle dynamics in turbulent channel flow. J. Turbul. 20 (7), 424-438.

Jiménez, J., Hoyas, S., Simens, M.P. \& Mizuno, Y. 2010 Turbulent boundary layers and channels at moderate Reynolds numbers. J. Fluid Mech. 657, 335-360.

Johansson, A.V. \& Alfredsson, P.H. 1982 On the structure of turbulent channel flow. J. Fluid Mech. 122, 295-314.

Jooss, Y., LI, L., BRACCHI, T. \& HEARST, R.J. 2021 Spatial development of a turbulent boundary layer subjected to freestream turbulence. J. Fluid Mech. 911, A4.

Kamruzzaman, M., Rømcke, O. \& Hearst, R.J. 2021 The impact of upstream turbulence on a plane jet. Exp. Fluids 62 (1), 18. 


\section{Asadi, M. Kamruzzaman and R. Jason Hearst}

Kim, J., Moin, P. \& Moser, R. 1987 Turbulence statistics in fully developed channel flow at low Reynolds number. J. Fluid Mech. 177, 133-166.

Kozul, M., Hearst, R.J., Monty, J.P., Ganapathisubramani, B. \& Chung, D. 2020 Response of the temporal turbulent boundary layer to decaying free-stream turbulence. J. Fluid Mech. 896, A11.

Krug, D., Philip, J. \& MARusic, I. 2017 Revisiting the law of the wake in wall turbulence. J. Fluid Mech. 811, 421-435.

Kwon, Y.S., Philip, J., De Silva, C.M., Hutchins, N. \& Monty, J.P. 2014 The quiescent core of turbulent channel flow. J. Fluid Mech. 751, 228-254.

Laskari, A., De Kat, R., Hearst, R.J. \& Ganapathisubramani, B. 2018 Time evolution of uniform momentum zones in a turbulent boundary layer. J. Fluid Mech. 842, 554-590.

LASKARI, A. \& MCKEON, B.J. 2021 Temporal characteristics of the probability density function of velocity in wall-bounded turbulent flows. J. Fluid Mech. 913, A6.

LeE, M. \& Moser, R.D. 2015 Direct numerical simulation of turbulent channel flow up to $R e_{\tau} \approx 5200$. J. Fluid Mech. 774, 395-415.

MakitA, H. 1991 Realization of a large-scale turbulence field in a small wind tunnel. Fluid Dyn. Res. 8 (1), 53-64.

MEINHART, C.D. \& ADRIAN, R.J. 1995 On the existence of uniform momentum zones in a turbulent boundary layer. Phys. Fluids 7 (4), 694-696.

MonTy, J.P. 2005 Developments in smooth wall turbulent duct flows. PhD thesis, University of Melbourne.

Monty, J.P., Hutchins, N., NG, H.C.H., Marusic, I. \& Chong, M.S. 2009 A comparison of turbulent pipe, channel and boundary layer flows. J. Fluid Mech. 632, 431-442.

Moser, R.D., Kim, J. \& MANsour, N.N. 1999 Direct numerical simulation of turbulent channel flow up to $R e_{\tau}=$ 590. Phys. Fluids 11 (4), 943-945.

Nagib, H.M. \& Chauhan, K.A. 2008 Variations of von Kármán coefficient in canonical flows. Phys. Fluids 20 (10), 101518.

Raffel, M., Willert, C.E., Scarano, F., Kähler, C.J., Wereley, S.T. \& Kompenhans, J. 2018 Particle Image Velocimetry: A Practical Guide. Springer.

RIND, E. \& CASTRO, I.P. 2012 On the effects of free-stream turbulence on axisymmetric disc wakes. Exp. Fluids 53 (2), 301-318.

Rodríguez-López, E., Bruce, P.J.K. \& BuXton, O.R.H. 2015 A robust post-processing method to determine skin friction in turbulent boundary layers from the velocity profile. Exp. Fluids 56 (4), 68.

Schultz, M.P. \& Flack, K.A. 2013 Reynolds-number scaling of turbulent channel flow. Phys. Fluids 25 (2), 025104.

Sciacchitano, A., Neal, D.R., Smith, B.L., Warner, S.O., Vlachos, P.P., Wieneke, B. \& SCARANO, F. 2015 Collaborative framework for PIV uncertainty quantification: comparative assessment of methods. Meas. Sci. Technol. 26 (7), 074004.

Sharp, N.S., NeUSCAmman, S. \& WARHAFT, Z. 2009 Effects of large-scale free stream turbulence on a turbulent boundary layer. Phys. Fluids 21 (9), 095105.

DE Silva, C.M., Hutchins, N. \& Marusic, I. 2016 Uniform momentum zones in turbulent boundary layers. J. Fluid Mech. 786, 309-331.

Smith, B.L. \& NeAL, D.R. 2016 Particle image velocimetry. In Handbook of Fluid Dynamics, 2nd edn. CRC Press.

Smits, A.J., McKeon, B.J. \& Marusic, I. 2011 High-Reynolds number wall turbulence. Annu. Rev. Fluid Mech. 43 (1), 353-375.

Teitel, M. \& AntoniA, R.A. 1990 The interaction region of a turbulent duct flow. Phys. Fluids A 2 (5), 808-813.

Thole, K.A. \& BogARD, D.G. 1996 High freestream turbulence effects on turbulent boundary layers. J. Fluids Engng 118 (2), 276-284.

Tuna, B.A., YARusevych, S., Li, X., Ren, Y. \& Shi, F. 2019 Investigation of the effect of inlet turbulence and Reynolds number on developing duct flow. J. Fluids Engng 141 (5), 051401.

Vinuesa, R., Noorani, A., Lozano-Durán, A., El Khoury, G.K., Schlatter, P., Fischer, P.F. \& NAGIB, H.M. 2014 Aspect ratio effects in turbulent duct flows studied through direct numerical simulation. J. Turbul. 15 (10), 677-706.

Vinuesa, R., Schlatter, P. \& NAGib, H.M. 2018 Secondary flow in turbulent ducts with increasing aspect ratio. Phys. Rev. Fluids 3 (5), 054606.

Wei, T. \& WillmaRTh, W.W. 1989 Reynolds-number effects on the structure of a turbulent channel flow. J. Fluid Mech. 204, 57-95.

WiENEKE, B. 2015 PIV uncertainty quantification from correlation statistics. Meas. Sci. Technol. 26 (7), 074002. 


\section{Effect of inlet turbulence on a channel's quiescent core}

Wu, X., Wallace, J.M. \& Hickey, J.-P. 2019 Boundary layer turbulence and freestream turbulence interface, turbulent spot and freestream turbulence interface, laminar boundary layer and freestream turbulence interface. Phys. Fluids 31 (4), 045104.

YANG, J., HWANG, J. \& SUNG, H.J. 2016 Structural organization of the quiescent core region in a turbulent channel flow. Intl J. Heat Fluid Flow 62, 455-463.

YANG, J., HwANG, J. \& SUNG, H.J. 2019 Influence of wall-attached structures on the boundary of the quiescent core region in turbulent pipe flow. Phys. Rev. Fluids 4 (11), 114606.

YOU, J. \& ZAKI, T.A. 2019 Conditional statistics and flow structures in turbulent boundary layers buffeted by free-stream disturbances. J. Fluid Mech. 866, 526-566.

YoU, J. \& ZAKI, T.A. 2020 Turbulent heat-transfer enhancement in boundary layers exposed to free-stream turbulence. Flow Turbul. Combust. 104 (2), 381-402. 